\title{
Spin Me Right Round Rotational Symmetry for FPGA-Specific AES
}

\author{
Lauren De Meyer ${ }^{1}$, Amir Moradi ${ }^{2}$ and Felix Wegener ${ }^{2}$ \\ ${ }^{1}$ imec - COSIC, KU Leuven, Belgium \\ lauren.demeyer@esat.kuleuven. be \\ ${ }^{2}$ Horst Görtz Institute for IT Security, Ruhr-Universität Bochum, Germany \\ firstname.lastname@rub.de
}

\begin{abstract}
The effort in reducing the area of AES implementations has largely been focused on Application-Specific Integrated Circuits (ASICs) in which a tower field construction leads to a small design of the AES S-box. In contrast, a naive implementation of the AES S-box has been the status-quo on Field-Programmable Gate Arrays (FPGAs). A similar discrepancy holds for masking schemes - a wellknown side-channel analysis countermeasure - which are commonly optimized to achieve minimal area in ASICs.

In this paper we demonstrate a representation of the AES S-box exploiting rotational symmetry which leads to a $50 \%$ reduction of the area footprint on FPGA devices. We present new AES implementations which improve on the state of the art and explore various trade-offs between area and latency. For instance, at the cost of increasing 4.5 times the latency, one of our design variants requires $25 \%$ less look-up tables (LUTs) than the smallest known AES on Xilinx FPGAs by Sasdrich and Güneysu at ASAP 2016. We further explore the protection of such implementations against first-order side-channel analysis attacks. Targeting the small area footprint on FPGAs, we introduce a heuristic-based algorithm to find a masking of a given function with $d+1$ shares. Its application to our new construction of the AES S-box allows us to introduce the smallest masked AES implementation on Xilinx FPGAs, to-date.
\end{abstract}

Keywords: AES - SCA · DPA - Rotational Symmetry - Threshold Implementations · $d+1$ Masking $\cdot$ FPGA

\section{Introduction}

Ever since the introduction of Differential Power Analysis (DPA) by Kocher et al. [KJJ99], protecting cryptographic devices against Side-Channel Analysis (SCA) has been a challenging and active area of research. A notable category of countermeasures is masking, in which a secret value is distributed among shares, which do not reveal any information about the secret separately. We speak of a $d^{\text {th }}$-order DPA attack when the adversary exploits the statistical moments of the SCA leakages (e.g., power consumption) up to order $d$. Such estimated statistical moments are expected to be independent of the secret, when sensitive variables are shared into $d+1$ shares.

In 2003, Ishai et al. [ISW03] introduced the $d$-probing model, in which a very powerful attacker has the ability to probe the exact values of up to $d$ intermediate variables. Security in this model has been related to more realistic adversary scenarios such as the noisy leakage [CJRR99] and the bounded moment leakage model [BDF $\left.{ }^{+} 17\right]$. However, in 2005 it was noted by Mangard et al. [MPO05] that the Boolean masking schemes which are secure in sequential platforms [Tri03, ISW03] still exhibit side-channel leakage when implemented 
in hardware. This is due to unintended transitions (or glitches) on wires before they stabilize. For hardware implementations, the probing model was therefore redefined using glitch-extended probes $\left[\mathrm{RBN}^{+} 15\right]$. The first masking scheme to achieve provable first-order security in the presence of glitches is Threshold Implementation (TI) [NRR06, NRS11], a particular realization of Boolean masking. As a result, the most challenging task in securing implementations is to mask the non-linear components of a cipher.

The AES S-box is an algebraically-generated vectorial Boolean function with 8-bit input and 8-bit output. It consists of an inversion in $\mathrm{GF}\left(2^{8}\right)$ followed by an affine transformation over $\mathrm{GF}(2)^{8}$. Having a small implementation of this S-box is important to achieve compact AES hardware, especially in the context of masked implementations. The tower field decomposition has proved to be a valuable approach to implement the field inversion, resulting in small AES S-boxes by Satoh et al. [SMTM01], Mentens et al. [MBPV05] and finally Canright [Can05]. One of those implementations exploits the rotation symmetry of a finite field inversion in $G F(16)$ [NRS08]. More recently, an even smaller S-box was created by Boyar et al. [BMP13] using a new logic optimization technique. This S-box implementation is the smallest to date. These S-box designs have all been successfully used to create the state-of-the-art smallest masked AES implementations $\left[\mathrm{BGN}^{+} 15, \mathrm{CRB}^{+} 16\right.$, GMK17, UHA17b]. However, when it comes to Look-up Table (LUT) based FPGA implementations, these optimized constructions do not perform better than the 8 slices that are required for any 8-bit to 8-bit mapping such as the AES S-box.

Another line of work in this area [Wam14, WHS15, WS17] exploits a property of inversion-based S-boxes that any inversion in $\operatorname{GF}\left(2^{n}\right)$ can be implemented by a Linear Feedback Shift Register (LFSR). The ASIC-based smallest such construction [Wam14] needs on average 127 clock cycles, i.e. its latency depends on the given S-box input, hence is vulnerable to timing attacks. The idea has been further developed in [WHS15] leading to 7 clock cycles latency (on average) for one S-box evaluation, which for sure needs more area compared to the original design. The authors also presented a constant-time variant of their design with a latency of 16 clock cycles. The underlying optimizations are not FPGA specific, and achieving SCA-protection by means of masking on such a construction does not seem easily possible ${ }^{1}$.

An FPGA design is indeed very different to its ASIC counterpart, most notably in the use of LUTs, which makes the number of inputs to a Boolean function a more defining factor for implementation cost than its algebraic complexity. Since the standardization of Rijndael as the AES, several successful efforts [CG03, $\left.\mathrm{BSQ}^{+} 08, \mathrm{CB} 12\right]$ have been made to reduce its size on FPGAs. In 2016, Sasdrich et al. [SG16] introduced an unprotected AES implementation on Xilinx Spartan-6 FPGAs which occupies 21 slices and remains the smallest FPGA implementation of AES known to date. Notably, in such a design the S-box is naively implemented as an 8-to-8 look-up table. The authors furthermore introduced a variant with 24 slices that additionally realizes shuffling as an SCA-hardening technique. Note that we exclude the designs like [CG03, $\left.\mathrm{NBD}^{+} 10, \mathrm{BGS}^{+} 11, \mathrm{BGD} 12, \mathrm{BDGH} 15\right]$ from our comparisons as their constructions relay on the Block RAM (BRAM) modules.

While research on masking mostly targets ASIC designs, some efforts have been made to utilize the specific architecture of an FPGA. In 2012, Moradi and Mischke [MM12] investigated a glitch-free implementation of masking on FPGAs by avoiding the occurrence of glitches with a special enable-logic, which has been further re-developed in [MW15] by Moradi and Wild. Sasdrich et al. [SMMG15] used the field-programmability to randomize the FPGA configuration during runtime. Recently, Vliegen et al. [VRM17] investigated the maximal throughput of masked AES-GCM on FPGAs. However, their masked Sbox is taken from $\left[\mathrm{MPL}^{+} 11\right]$ without further FPGA-specific improvements. We would

\footnotetext{
${ }^{1}$ It is based on the fact that every $x \in \operatorname{GF}\left(2^{8}\right)$ is presented by $\alpha^{n}$ and its inverse by $\left(\alpha^{-1}\right)^{n}$. So, two LFSRs constantly multiply by $\alpha$ and $\alpha^{-1}$. When one of them reaches $x$, the other one is $x^{-1}$. The concept does not work when $x$ is shared by Boolean masking.
} 
like to emphasize that several AES masked FPGA designs have been reported in the literature which consider neither the glitches nor the non-completeness property defined in TI [NRS11]. For example, the masked S-box design used in [RWS11] is not different to Canright and Batina's design [CB08] which has been shown to have first-order exploitable leakage [MPO05, MME10].

Our Contribution. In this work, we exclusively focus on FPGA devices and in particular those of Xilinx. All our case studies target a Xilinx Spartan-6 FPGA. We exploit a rotational symmetry property of Galois field power maps, e.g. the field inversion, to construct a novel structure realizing the AES S-box. This leads to an FPGA footprint of only 4 slices which is - to the best of our knowledge - smaller than any reported FPGA-based design of the AES S-box in the literature. Such an area reduction comes at the cost of a latency of 8 clock cycles for one S-box evaluation. We present several new AES implementations for Xilinx FPGAs. We adapt the currently smallest known FPGA-based AES design of [SG16] to use our S-box construction and achieve a new design that occupies only 17 slices - a $19 \%$ reduction over the previous record. We also restructure the smallest known ASIC-based AES design of [JMPS17] to efficiently use the FPGA resources and combine it with our S-box design, leading to another very small footprint of only 63 LUTs for the entire encryption function. Our designs use only FPGA LUTs and other slice-internal components such as slice registers and internal MUXes, but no block RAM (BRAM) which has been used in $\left[\mathrm{BGS}^{+} 11, \mathrm{NBD}^{+} 10, \mathrm{BDGH} 15, \mathrm{BGD} 12\right]$ as a principle feature.

In the second part of this work, we extend our construction for SCA resistance. To this end, we apply Boolean masking with a minimum number of two shares on a decomposition of the AES S-box, which again exploits the rotational symmetry. We detail a heuristic optimization methodology for finding a non-complete masking of non-quadratic Boolean functions. Our approach targets a minimum number of input variables per component, which is suitable for a compact realization in the LUT-based structure of FPGAs. Targeting an optimized implementation with respect to LUT utilization, we introduce - to the best of our knowledge - the smallest masked AES design on Xilinx FPGAs. We deploy our design on a Spartan- 6 and evaluate its SCA resistance by practical experiments.

\section{Preliminaries}

In the following we give an introduction to FPGA technology, Boolean algebra and masking schemes to counteract SCA attacks. Further, we define the notation for the rest of the paper.

\section{1 $\quad$ FPGAs}

FPGAs are reconfigurable hardware devices consisting of configurable logic blocks (CLB), each of which is further subdivided into two slices that each contain four look-up tables (LUTs), eight registers and additional carry-logic. In the following, we give a bottom-up description of the the structure of Xilinx Spartan-6 FPGAs, but this is similar for series 7 devices and FPGAs of other manufacturers.

\subsubsection{LUTs}

An FPGA's LUT is a combination of a multiplexer tree and read-only RAM. The Xilinx 6 and 7 series contain one type of LUT block, which can be used to create functions with either six input bits and one output bit (O6) or five input bits and two output bits (O6,O5). This is illustrated in Figure 1a. 


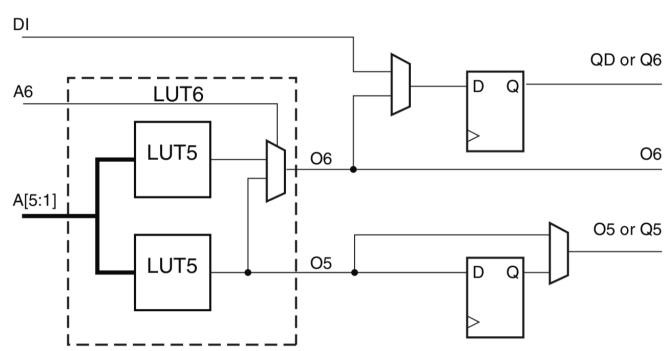

(a) Spartan-6 LUT block

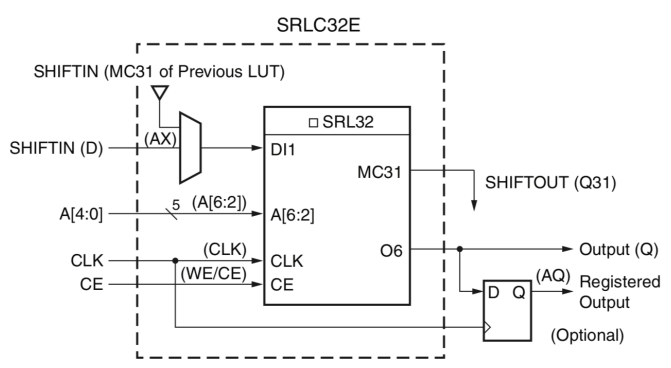

(b) LUT as a 32-bit shiftregister

Figure 1: The illustrations are taken from [Xil10].

Because of this structure, the algebraic complexity of Boolean functions does not matter in FPGAs as long as the number of inputs is six or fewer. When realizing a vectorial Boolean function on FPGAs, two coordinates that jointly depend on five or fewer inputs can be mapped into one LUT. This puts FPGA design in stark contrast with ASIC design as they clearly demand very different optimization strategies to achieve a low-cost implementation.

There are alternative uses to the circuitry of a LUT. A single LUT $^{2}$ can also be configured as a 32-bit shift register with a 5-bit read address port in addition to serial shiftin and shiftout ports (see Figure 1b). It is also possible for a LUT to be used as 32 addressable RAM cells of two bits each.

\subsubsection{Slices}

When mapping a hardware design to an FPGA, we count the number of occupied slices as a metric for size. As each slice contains not only four LUTs but also further logic gates and registers, this opens up more optimization potential compared to a naive mapping to LUTs exclusively.

More Inputs. Since each slice consist of four LUTs, it can trivially realize four 6-to-1bit functions. Further, due to internal multiplexers between the four LUTs, each slice can also implement two 7-to-1-bit functions or one 8-to-1-bit function. As a result, the 8-bit AES S-box can be easily implemented in 8 slices; one for each Boolean coordinate function. In fact, this is the smallest known FPGA implementation of the AES S-box, used in $\left[\mathrm{BSQ}^{+} 08, \mathrm{SG} 16\right]$.

Memory. A slice also contains eight flip-flops, connected to the O5 and O6 output of each LUT (see Figure 1a). Note that every slice is limited in its functionality by many constraints. For example, while the inputs to four of the eight registers are directly accessible from the slice-external wires, a connection to the other four can only be made via the LUTs.

Types. In Spartan-6 devices we distinguish three different types of slices: The SliceX contains only four LUTs and eight flip-flops, while the SliceL contains additional carry logic and finally the most complex one, SliceM, can be used as a RAM unit with 256 bits of memory in different chunks of addressability or a 128-bit shift register.

\footnotetext{
${ }^{2}$ Only in particular slice type SliceM.
} 


\subsection{Mathematical Foundations}

Boolean Algebra. We define $(\mathrm{GF}(2),+, \cdot)$ as the field with two elements ZERo and ONE. We denote the $n$-dimensional vector space defined over this field by $\operatorname{GF}(2)^{n}$. Its elements can be represented by $n$-bit numbers and added by bit-wise XOR. In contrast, the Galois Field $\operatorname{GF}\left(2^{n}\right)$ contains an additional field multiplication operation. It is well known that $\mathrm{GF}(2)^{n}$ and $\mathrm{GF}\left(2^{n}\right)$ are isomorphic.

A Boolean function $F$ is defined as $F: \operatorname{GF}(2)^{n} \rightarrow \mathrm{GF}(2)$, while we call $G: \operatorname{GF}(2)^{n} \rightarrow$ $\mathrm{GF}(2)^{n}$ a vectorial Boolean function. A (vectorial) Boolean function can be represented by a look-up table (LUT), which is a list of all output values for each of the $2^{n}$ input combinations. Further, each Boolean function can be described by a unique representation - so called normal form. Most notably the Algebraic Normal Form (ANF) is the unique representation of a Boolean function as a sum of monomials. In this work, we designate by $m \in \operatorname{GF}\left(2^{n}\right)$ the monomial $x_{0}^{m_{0}} x_{1}^{m_{1}} \ldots x_{n-1}^{m_{n-1}}$ where $\left(m_{0}, m_{1}, \ldots, m_{n-1}\right)$ is the bitvector of $m$. The monomial's algebraic degree is simply its hamming weight: $\operatorname{deg}(m)=\mathrm{hw}(m)$. We can then write the ANF of any Boolean function $F$ as

$$
F(x)=\bigoplus_{m \in \operatorname{GF}\left(2^{n}\right)} a_{m} x_{0}^{m_{0}} x_{1}^{m_{1}} \ldots x_{n-1}^{m_{n-1}}
$$

The algebraic degree of $F$ is the largest number of inputs occurring in a monomial with a non-zero coefficient:

$$
\operatorname{deg}(F)=\max _{m \in \operatorname{GF}\left(2^{n}\right), a_{m} \neq 0} \operatorname{hw}(m)
$$

Finite Field Bases. We denote the isomorphism between the finite field $\operatorname{GF}\left(2^{n}\right)$ and the vector space $\mathrm{GF}(2)^{n}$ by $\phi: \mathrm{GF}\left(2^{n}\right) \rightarrow \mathrm{GF}(2)^{n}$. This mapping depends on the basis chosen for $\mathrm{GF}\left(2^{n}\right)$. The vector $\phi(x)=\left(a_{0}, \ldots, a_{n-1}\right) \in \mathrm{GF}(2)^{n}$ holds the coordinates of $x$ with respect to that basis, and we denote by $\phi(x)_{i}$ the $i^{\text {th }}$ coordinate of this vector. A polynomial basis has the form

$$
\left(1, \alpha, \alpha^{2}, \ldots, \alpha^{n-1}\right)
$$

with $\alpha \in \mathrm{GF}\left(2^{n}\right)$ the root of a primitive polynomial of degree $n$. We denote $\phi^{\alpha}$ the isomorphism mapping to a polynomial basis with $\alpha$.

Consider for example $\alpha=2$. In that case, we have $\phi^{2}\left(2^{i}\right)=\mathbf{e}_{i}$ with $\mathbf{e}_{i}$ the $i^{\text {th }}$ unit vector, so the representation of $x \in \mathrm{GF}\left(2^{n}\right)$ in polynomial basis simply corresponds to its binary expansion.

In contrast, a normal basis has the form

$$
\left(\beta^{2^{0}}, \beta^{2^{1}}, \ldots, \beta^{2^{n-1}}\right)
$$

with $2^{n-1}$ possible choices for $\beta \in \mathrm{GF}\left(2^{n}\right)$. In a normal basis over any finite field, the zero (resp. unit) element is represented by a coordinate vector of all zeros (resp. all ones). An element $\beta \in \mathrm{GF}\left(2^{n}\right)$ can thus form a normal basis if $\bigoplus_{i=0}^{n-1} \beta^{2^{i}}=1$. We denote by $\phi_{n}^{\beta}(x)$ the isomorphic mapping from $x \in \mathrm{GF}\left(2^{n}\right)$ to its $\mathrm{GF}(2)^{n}$ representation in normal basis with $\beta$, although we sometimes omit $\beta$ for ease of notation.

The conversion between any polynomial and normal basis is merely a linear transformation which can be represented by a matrix multiplication over $\mathrm{GF}(2)^{n}$. The matrix can be determined column-wise by mapping each basis element of the original basis to the target basis. Let $Q \in \mathrm{GF}(2)^{n \times n}$ be the matrix mapping from a normal basis with $\beta$ to a polynomial basis with $\alpha$, i.e. $Q \times \phi_{n}^{\beta}(x)=\phi^{\alpha}(x)$. Then, the $i^{\text {th }}$ column of $Q$ is simply $\phi^{\alpha}\left(\beta^{2^{i}}\right)$. The inverse mapping uses the inverse matrix: $Q^{-1} \times \phi^{\alpha}(x)=\phi_{n}^{\beta}(x)$. 


\subsection{Boolean Masking in Hardware}

We denote the $s_{i}$-sharing of a secret variable $x$ as $\boldsymbol{x}=\left(x_{0}, \ldots, x_{s_{i}-1}\right)$ and similarly an $s_{o}$-sharing of a Boolean function $F(x)$ as $\boldsymbol{F}=\left(F_{0}, \ldots, F_{s_{o}-1}\right)$. Each component function $F_{i}$ computes one share $y_{i}$ of the output $y=F(x)$. A correctness property should hold for any Boolean masking:

$$
x=\bigoplus_{0 \leq j<s_{i}} x_{j} \Leftrightarrow F(x)=\bigoplus_{0 \leq j<s_{o}} F_{j}(\boldsymbol{x})
$$

We define $\mathcal{S}(x)$ as the set of all correct sharings of the value $x$. Creating a secure masking of cryptographic algorithms in hardware is especially challenging due to glitches. Despite this major challenge, Nikova et al. [NRR06] introduced a provably secure scheme against first-order SCA attacks in the presence of glitches, named Threshold Implementation (TI). A key concept of TI is the non-completeness property which we recall here.

Definition 1 (Non-Completeness). A sharing $\boldsymbol{F}$ is non-complete if any component function $F_{i}$ is independent of at least one input share.

Apart from non-completeness, the security proof of TI depends on a uniform distribution of the input sharing fed to a shared function $\boldsymbol{F}$. For example, when considering round-based block ciphers, the output of one round serves as the input of the next. Hence, a shared implementation of $F$ needs to maintain this property of uniformity.

Definition 2 (Uniformity). A sharing $\boldsymbol{x}$ of $x$ is uniform, if it is drawn from a uniform probability distribution over $\mathcal{S}(x)$.

We call $\boldsymbol{F}$ a uniform sharing of $F(x)$, if it maps a uniform input sharing $\boldsymbol{x}$ to a uniform output sharing $\boldsymbol{y}$ :

$$
\exists c: \forall x \in \mathrm{GF}(2)^{n}, \forall \boldsymbol{x} \in \mathcal{S}(x), \forall \boldsymbol{y} \in \mathcal{S}(F(x)): \operatorname{Pr}(\boldsymbol{F}(\boldsymbol{x})=\boldsymbol{y})=c .
$$

Finding a uniform sharing without using fresh randomness is often tedious $\left[\mathrm{BNN}^{+} 12\right.$, BB16] and may be impossible. Hence, many masking schemes restore the uniformity by re-masking with fresh randomness. When targeting first-order security, one can re-mask $s$ output shares with $s-1$ shares of randomness as such:

$$
\left(F_{0} \oplus r_{0}, F_{1} \oplus r_{1}, \ldots, F_{s-2} \oplus r_{s-2}, F_{s-1} \oplus \bigoplus_{0 \leq j \leq s-2} r_{j}\right)
$$

Threshold Implementation was initially defined to need $s_{i} \geq t d+1$ shares with $d$ the security order and $t$ the algebraic degree of the Boolean function $F$ to be masked. The non-completeness definition was extended to the level of individual variables in $\left[\mathrm{RBN}^{+} 15\right]$, which allowed the authors to reduce the number of input shares to $s_{i}=d+1$, regardless of the algebraic degree. As a result, the number of output shares $s_{o}$ increases to $(d+1)^{t}$. For example, two shared secrets $\boldsymbol{a}=\left(a_{0}, a_{1}\right)$ and $\boldsymbol{b}=\left(b_{0}, b_{1}\right)$ can be multiplied into a 4 -share $\boldsymbol{c}=\left(c_{0}, c_{1}, c_{2}, c_{3}\right)$ by just computing the cross products.

$$
\begin{array}{ll}
c_{0}=a_{0} b_{0} & c_{1}=a_{0} b_{1} \\
c_{2}=a_{1} b_{0} & c_{3}=a_{1} b_{1}
\end{array}
$$

The number of output shares can be compressed back to $d+1$ after a refreshing and a register stage. This method was first applied to the AES S-box in $\left[\mathrm{CRB}^{+} 16\right]$ and lead to a reduction in area, but an increase in the randomness cost.

A similar method for sharing 2-input AND gates with $d+1$ shares is demonstrated by Gross et al. in [GMK16, GMK17]. In particular, they propose to refresh only the cross-domain products $a_{i} b_{j}$ for $i \neq j$, resulting in a fresh randomness cost of $\left(\begin{array}{c}d+1 \\ 2\end{array}\right)$ units. 
In [UHA17a], Ueno et al. demonstrate a general method to find a $d+1$-sharing of a non-quadratic function with $d+1$ input shares in a non-complete way by suggesting a probabilistic heuristic that produces $(d+1)^{n}$ output shares in the worst case, where $n$ stands for the number of variables.

\section{Rotational Symmetry of the AES S-box}

Rotational Symmetry of Power Maps. In 2008, Rijmen et al. [RBF08] noted a rotational property of power maps in finite fields. More specifically, they showed that every power map based S-box (or vectorial Boolean function) over $\mathrm{GF}\left(2^{n}\right)$ is a rotation-symmetric S-box in a normal basis. We denote by $\operatorname{rot}(v, i)$ the $i$-times rotation of $v \in \operatorname{GF}(2)^{n}$ to the right, i.e. $\operatorname{rot}(v, 1)=\left(a_{n-1}, a_{0}, \ldots, a_{n-2}\right)$ when $v=\left(a_{0}, a_{1}, \ldots, a_{n-1}\right)$. When $i$ is omitted, it is equal to 1 .

Definition 3 (Rotation-Symmetry). An $n$-bit S-box $S: \mathrm{GF}(2)^{n} \rightarrow \mathrm{GF}(2)^{n}$ is rotationsymmetric if and only if $\operatorname{rot}(S(v))=S(\operatorname{rot}(v))$ for all $v \in \mathrm{GF}(2)^{n}$.

We consider a normal basis with $\beta$ :

$$
\left(\beta_{0}, \beta_{1}, \beta_{2}, \ldots, \beta_{n-1}\right)=\left(\beta, \beta^{2}, \beta^{2^{2}}, \ldots, \beta^{2^{n-1}}\right)
$$

This basis allows for an effective realization of squaring. As the order of the multiplicative group is $2^{n}-1$, we derive that $\forall x \in \mathrm{GF}\left(2^{n}\right): x^{2^{n}-1}=1$ by Lagrange's theorem. As a result, we have that $x^{2^{n}}=x$ for any element in $\operatorname{GF}\left(2^{n}\right)$. This leads to the following lemma.

Lemma 1 ([RBF08]). In a normal basis over $\mathrm{GF}\left(2^{n}\right)$, the squaring operation corresponds to a rotation of the coordinates vector: $\phi_{n}\left(x^{2}\right)=\operatorname{rot}\left(\phi_{n}(x)\right)$

Proof. We make use of the fact that $x=x^{2^{n}}$ holds for any element in $\operatorname{GF}\left(2^{n}\right)$.

$$
\begin{aligned}
x^{2} & =a_{0} \beta_{0}^{2}+a_{1} \beta_{1}^{2} \ldots+a_{n-2} \beta_{n-2}^{2}+a_{n-1} \beta_{n-1}^{2} \\
& =a_{0} \beta^{2}+a_{1} \beta^{2^{2}} \ldots+a_{n-2} \beta^{2^{n}-1}+a_{n-1} \beta^{2^{n}} \\
& =a_{n-1} \beta+a_{0} \beta^{2}+a_{1} \beta^{2^{2}} \ldots+a_{n-2} \beta^{2^{n-1}} \\
& =a_{n-1} \beta_{0}+a_{0} \beta_{1}+a_{1} \beta_{2} \ldots+a_{n-2} \beta_{n-1}
\end{aligned}
$$

Hence, the below equation holds.

$$
\phi_{n}\left(x^{2}\right)=\left(a_{n-1}, a_{0}, \ldots, a_{n-2}\right)=\operatorname{rot}\left(\phi_{n}(x), 1\right)
$$

Successive application of the above property yields the relation

$$
\phi_{n}\left(x^{2^{i}}\right)=\operatorname{rot}\left(\phi_{n}(x), i\right)
$$

Now consider a power map $F(x)=x^{k}$ over $G F\left(2^{n}\right)$. Clearly, for any power map we have that $F(x)^{l}=F\left(x^{l}\right)$. Let $S\left(\phi_{n}(x)\right)=\phi_{n}(F(x))$ be the normal basis S-box over $\operatorname{GF}(2)^{n}$ for which $F(x)$ is an algebraic description. We denote the component Boolean functions by $S_{i}: \operatorname{GF}(2)^{n} \rightarrow \mathrm{GF}(2)$. By Theorem 9 in [RBF08], $S$ is thus rotation-symmetric, i.e. $\operatorname{rot}(S(v))=S(\operatorname{rot}(v))$ for all $v \in \mathrm{GF}(2)^{n}$ or equivalently, for each $i \in\{0, \ldots, n-1\}$ : $S_{i}(v)=S_{0}(\operatorname{rot}(v, i))$. All $n$ output bits of the S-box can be calculated using the same Boolean function $S_{0}$. From now on, we denote the Boolean function that calculates the least significant bit of the S-box output as $S^{*}(v)=S_{0}(v)$. It is related to the power map function 
as follows: $S^{*}\left(\phi_{n}(x)\right)=\phi_{n}(F(x))_{0}$. We demonstrate the rotational symmetry and show how to calculate the $i^{\text {th }}$ coordinate of the power map's normal basis representation:

$$
\begin{aligned}
S_{i}\left(\phi_{n}(x)\right)=\phi_{n}(F(x))_{i} & =\operatorname{rot}\left(\phi_{n}\left(F(x)^{2^{i}}\right),-i\right)_{i} \\
& =\operatorname{rot}\left(\phi_{n}\left(F(x)^{2^{i}}\right), 0\right)_{0} \\
& =\phi_{n}\left(F(x)^{2^{i}}\right)_{0} \\
& =\phi_{n}\left(F\left(x^{2^{i}}\right)\right)_{0} \\
& =S^{*}\left(\phi_{n}\left(x^{2^{i}}\right)\right) \\
& =S^{*}\left(\operatorname{rot}\left(\phi_{n}(x), i\right)\right)
\end{aligned}
$$

Note that $\phi_{n}$ and by extension $S^{*}$ depend on the choice of $\beta$, which generates the normal basis, but we omit $\beta$ here for readability.

As a result, instead of $n$ Boolean functions $S_{0}, S_{1}, \ldots, S_{n-1}$ operating in parallel, the power map based S-box $S$ can be evaluated entirely with a single $n$-to-1-bit function $S^{*}$ by rotating the input vector bitwise.

\section{Smallest Unprotected AES on FPGAs}

It is generally known that an optimal FPGA implementation of the AES S-box requires 32 LUTs in eight slices, as each of its eight coordinate functions is an 8-to-1 mapping (see Section 2.1.2). There is no obvious way to reduce this number, as every linear combination of coordinate functions maintains the maximal algebraic degree of seven and depends on all eight inputs. Hence, every coordinate function occupies an entire slice.

Note that Canright's tower field construction [Can05] does not provide an alternative as it is ill-suited for Spartan-6 devices due to the underutilization of six-input LUTs by the operations in $\operatorname{GF}\left(2^{4}\right)$ and even $\mathrm{GF}\left(2^{2}\right)$. More precisely, realizing the basis conversion, square-scaling, inversion and multiplications can occupy as much as 53 LUTs on an FPGA.

\subsection{Optimizing the S-box for FPGA}

S-box Structure. We demonstrate that it is indeed possible to realize the AES S-box in fewer LUTs by trading off latency for area. Recall that the AES S-box consists of an inversion in $\operatorname{GF}\left(2^{8}\right)$, followed by an affine transform over $\operatorname{GF}(2)^{8}$. For the inversion part, we exploit the rotational symmetry of the power map $x^{254}$ in $\operatorname{GF}\left(2^{8}\right)$ as explained in Section 3. The structure is illustrated in Figure 2a. Since the AES inversion is defined in a polynomial basis with $\alpha=2$, we first convert the input byte $x$ to a normal basis using a linear transform ("p2n"). Then, in a bit-wise fashion, we calculate the output of the rotation-symmetric S-box by rotating the first register R1. The single-bit output of $S^{*}$ is shifted into a second register R2. When all eight bits have been calculated, we use another linear transform to convert the result back into the polynomial basis ("n2p"). This transform is combined with the affine transform of the AES S-box.

S-box Implementation Cost. We examine various normal bases and target a minimal number of LUTs needed to implement the 8-to-8-bit functions p2n and n2p. Note that it is not required to optimize $S^{*}$ since it is an 8-to-1-bit Boolean function of algebraic degree 7 and requires 4 LUTs (an entire slice) in any normal basis. At the end of our investigations, we pick $\beta=145 .^{3}$ By optimizing our implementation for intensive usage of 5-to-2 LUTs,

\footnotetext{
${ }^{3}$ The algebraic normal forms for $S^{*}, \mathrm{p} 2 \mathrm{n}$ and $\mathrm{n} 2 \mathrm{p}$ are given in Appendix A
} 


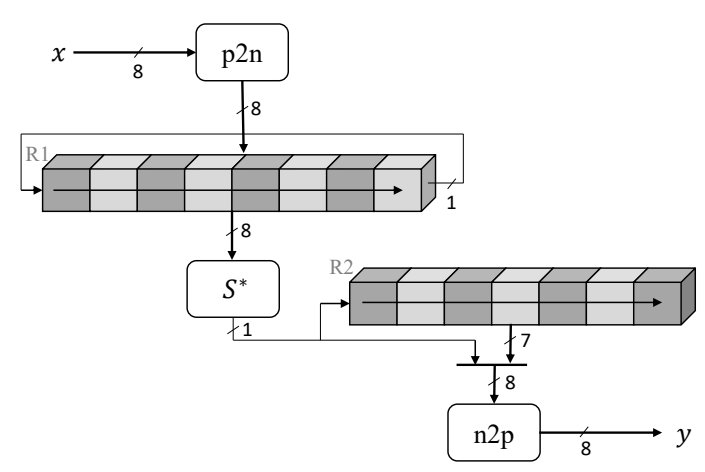

(a) byte-parallel loading

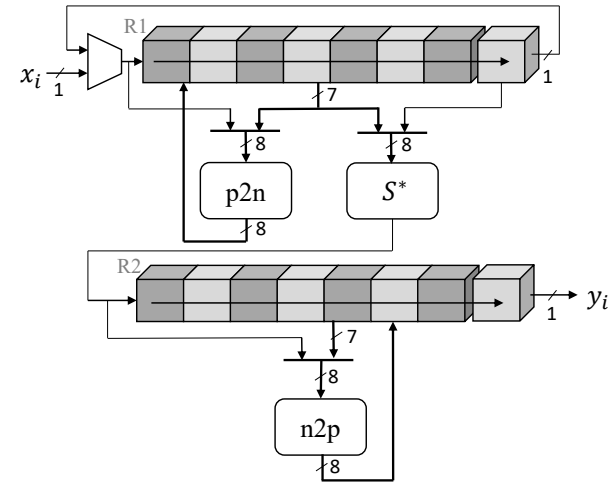

(b) bit-serial loading

Figure 2: Illustration of the bit-serial AES S-box based on rotational symmetry.

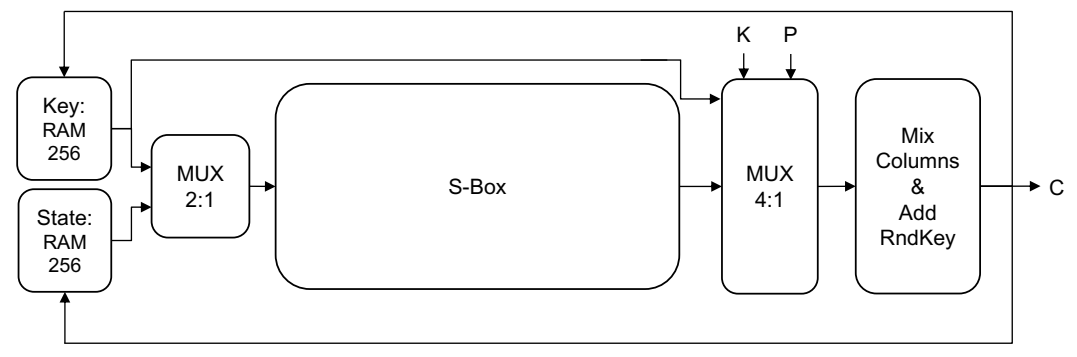

Figure 3: Illustration of the byte-wise AES design by [SG16]. All wires are 8-bit wide. Especially notable is the 8-bit aggregation register in the MixColumns block. The RAM blocks are further divided into two parts of 128 bits which are used in alteration.

we can implement the affine transformations $\mathrm{p} 2 \mathrm{n}$ and $\mathrm{n} 2 \mathrm{p}$ and the rotating register $\mathrm{R} 1 \mathrm{in}$ one slice each. More specifically, the affine transforms each consume 4 LUTs. The 8-bit register R1 uses all 8 registers in a slice. The choice between parallel loading and rotational shifting is achieved using the four LUTs of that slice. As mentioned previously, $S^{*}$ itself also occupies 1 slice. Finally, the 7 slice flip-flops for R2 are found in the already used slices for $\mathrm{n} 2 \mathrm{p}, \mathrm{p} 2 \mathrm{n}$ and $S^{*}$. In total, the S-box design occupies 16 LUTs and 15 registers, all fitting into only 4 slices. This means a $50 \%$ reduction over the status-quo [BSQ $\left.{ }^{+} 08, \mathrm{SG} 16\right]$.

We pay for the reduction in area with latency. While the 32-LUT S-box computes the output within one clock cycle, our bit-serialized approach (Figure 2a in 16 LUTs) increases the latency to 8 clock cycles. The linear function $\mathrm{p} 2 \mathrm{n}$ is applied immediately to the S-box input $x$. In cycles 1 to 8 , register R1 rotates while $S^{*}$ serially computes each output bit. The outputs are shifted into R2 bit by bit. In the last cycle, the last output bit is combined with the 7-bit content of R2 as input to the affine transform n2p, which computes the S-box output $y$. The register bypassing of $\mathrm{n} 2 \mathrm{p}$ allows the S-box latency to be 8 cycles and the $\mathrm{R} 2$ register to be only 7 bits wide.

\subsection{Fully Byte-serial AES}

A Grain in the Silicon. We start from the smallest unprotected state-of-the-art AES design for FPGA [SG16] illustrated in Figure 3. The entire implementation requires only 21 slices, of which 15 slices construct the round function and key schedule, including 8 
Table 1: Overview of unprotected AES implementations for FPGA

\begin{tabular}{lccccc}
\hline Design & \# LUTs & \# Flip flops & \# Slices & \# Clocks & $\mathbf{f}_{\text {max }}{ }^{4}$ \\
\hline Sasdrich et al. [SG16] & 84 & 24 & 21 & 1471 & $108 \mathrm{MHz}$ \\
Latency optimized & 81 & $\mathbf{2 1}$ & 21 & $\mathbf{1 0 9 8}$ & $113 \mathrm{MHz}$ \\
With bit-serial S-box & 68 & 39 & $\mathbf{1 7}$ & 5538 & $109 \mathrm{MHz}$ \\
Fully bit-serialized & $\mathbf{6 3}$ & 38 & 19 & 4852 & $\mathbf{1 5 5} \mathbf{~ M H z}$ \\
\hline
\end{tabular}

slices for the AES S-box and 2 slices configured as 256-bit memory for the state and key arrays. The round constants are also stored in this memory. The remaining 6 slices make up a heavily optimized control unit with a finite state machine (FSM) of 32 states. Each round in this design requires 147 clock cycles. In the first 50 cycles, the key schedule is performed to compute the entire 128-bit key state of the current round. In the next 97 cycles the round function is computed, using the freshly calculated round key. Most of these clock cycles is spent on the MixColumns operation because it performs 4 S-box evaluations on the fly for each byte of the MixColumns output. The S-box outputs are not stored but discarded and recomputed when needed. Therefore, 64 S-box invocations (instead of 16) are performed. In the last round, MixColumns is omitted and the round function takes only 33 clock cycles. With 65 cycles spent on loading a new plaintext and key, an entire encryption has a latency of $(65+(50+97) \times 9+50+33)=1471$ clock cycles. For more details on this design, we refer to the original work [SG16].

Latency optimization. We note that the above design can be optimized with respect to latency without sacrificing its minimal area requirement. Instead of performing the key schedule and round function separately in each round, we can interleave them, i.e. we compute one key byte and immediately use it to update the corresponding state byte. To do this, we only have to adapt the control logic. We create a new FSM of 16 states and derive the LUT mappings for the control signals and addresses. We decrease the number of LUTs from 24 to 21 and the number of flip flops from 16 to 13 . The resulting design has a latency of 113 clock cycles per round, except 49 in the last round. Loading of plaintext and key bytes is done in 32 cycles. In total, one encryption requires $(32+113 \times 9+49)=1098$ clock cycles. Note that this design retains the original 8-LUT S-box. It is summarized in row 2 of Table 1.

Bit-serializing the S-box. We now start from the latency-optimized design and replace the 8-slice byte-parallel S-box with our bit-serialized S-box from Figure 2a. We accordingly change the control unit to make use of such an S-box design by means of an extra 3-bit counter to account for the S-box latency. It still contains an FSM of 16 states. This results once again in a control unit of 24 LUTs and 16 flip flops. Each cipher round now has a latency of 589 clock cycles and the last round 205 cycles. Hence, one encryption is completed in $(32+589 \times 9+205)=5538$ clock cycles. An overview of the post-map area and latency of this designs is shown in row 3 of Table 1 . We can fit the entire AES encryption into only 17 slices, a $19 \%$ reduction over the state-of-the-art.

\subsection{Fully Bit-serial AES}

We now combine our bit-serialized AES S-box with the bit-serialized AES implementation of [JMPS17]. We first adopt the S-box for bit-serial loading and then we adopt their AES design for FPGAs, since it originally targets ASIC platforms.

\footnotetext{
${ }^{4}$ From the Post-PAR Static Timing Report
} 
S-box. The structure of the bit-serialized S-box with bit-serial loading is shown in Figure $2 \mathrm{~b}$. The conversions to and from the normal basis (p2n and n2p modules) are now realized in 12 LUTs, i.e. 3 slices (including the S-box affine). This is more than before because these LUTs also implement the choice between the parallel and shift-serial input to R1 and R2. This new constraint requires a different normal basis than before to achieve the stated size. We choose $\beta=133 .{ }^{5}$ As a result, shift-registers $\mathrm{R} 1$ and $\mathrm{R} 2$ only require 16 more flip-flops, for which we can use the same slices. The 8-to-1-bit Boolean function $S^{*}$ still occupies exactly 4 LUTs of a slice. Therefore, the entire S-box circuit, i.e. all elements and components shown in Figure 2b, requires only 16 LUTs and 16 flip-flops fitting into 4 slices (again 50\% less area compared to [SG16]).

The S-box now has a latency of 16 cycles. In cycles 1 to 7 , input bits are shifted into the first register. In cycle 8 , the linear conversion $\mathrm{p} 2 \mathrm{n}$ is applied to the 7 -bit content of the register and the newest incoming bit at input $x_{i}$. The 8 -bit result is written to that same register in parallel in the same cycle. In the 8 subsequent cycles (9 to 16), this register is rotated, which allows $S^{*}$ to evaluate the 8-bit output. The first 7 bits are shifted serially into R2. In cycle 16 , the affine conversion $\mathrm{n} 2 \mathrm{p}$ is applied to the seven bits stored in R2 and the last output of $S^{*}$. The result is written in parallel to R2. The AES S-box output $y$ is then ready to be shifted out serially over eight cycles. Note that this can be done in parallel with the feeding of the next S-box input into R1.

Architecture. Our design is shown in Figure 4. We refer to [JMPS17, Fig. 3,4] for the corresponding original architecture. To accommodate for bit-sliding, we instantiate four LUTs as 32-bit shift registers (SRLC32E, see Figure 1b) for both the state and key arrays. Each LUT represents one row of the array and has its own shift enable signal (not drawn). This means that ShiftRows can be implemented without additional area cost by letting row $i \in\{0,1,2,3\}$ shift $8 i$ times. This requires 24 clock cycles in total. As shown in Figure 1b, the shift register LUT has both a serial output and a custom read port. In the state array, this port reads the next-to-last bit, which is used in the computation of MixColumns. In the key array, this port reads the $7^{\text {th }}$ bit of each row. The MixColumns is performed in 32 clock cycles as in [JMPS17]. The implementation uses 6 LUTs and 4 flip flops (for the four most significant bits). We plug in the 16-LUT S-box as described in Section 4.1. With a bit-serial loading of the input, the S-box has a latency of 16 clock cycles. The same S-box is shared between the round function and key schedule. The multiplexers in the state array can be implemented using 4 LUTs. The same goes for the operations at the input of each row of the key state. We also have one LUT for the AddRoundKey which also includes two multiplexers to select the serial input to R1. On the one hand, it chooses $x_{i}$ between the S-box input from the round function and from the key schedule. On the other hand, it chooses the feedback from $\mathrm{R} 1$ when $\mathrm{R} 1$ should be rotating, i.e. the multiplexer shown in Figure $2 b$.

Finally, we make a controller to supply the control signals, read addresses and round constant to the round function, key schedule and S-box. The controller consists of an FSM with 8 states, which are encoded in a way that minimizes the number of LUTs needed to compute the control signals and addresses. In total, the control unit takes up 24 LUTs and 18 flip flops. This brings the total LUT cost of the AES implementation on a new record of 63 LUTs (see Table 1, row 4). The bit-serial loading of plaintext and key requires 128 clock cycles. Each encryption round is done in 476 cycles, except the last round, which is done in 440 cycles. In total, one encryption takes $(128+476 \times 9+440)=4852$ clock cycles. It might be surprising that this bit-serialized design is faster than the byte-serialized AES from Section 4.2. This is due to the high latency of the S-box and the fact that the architecture of [SG16] has a "wasteful" MixColumns implementations that evaluates the S-box multiple times.

\footnotetext{
${ }^{5}$ The algebraic normal form for $S^{*}, \mathrm{p} 2 \mathrm{n}$ and $\mathrm{n} 2 \mathrm{p}$ are given in Appendix B
} 

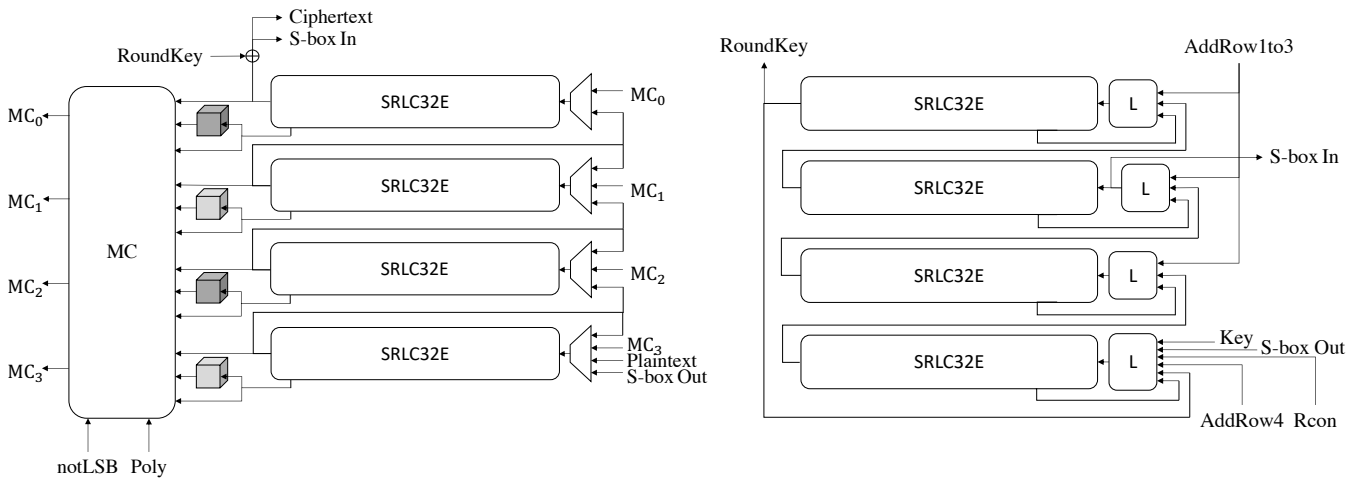

Figure 4: Bit-serial architecture for AES-128. Left: State Array and Round Function, Right: Key Schedule

\section{Masked AES}

The rotational symmetry approach to implement the AES S-box reduces its non-linear proportion significantly. This is especially interesting when we consider the application of masking schemes. It is well known that the non-linear parts of a circuit grow exponentially in masked implementations, while linear operations can simply be duplicated and performed on each share independently, i.e. a linear increase in the area. Instead of sharing a complete 8-bit to 8-bit mapping, the rotational symmetry approach allows us to mask only a single 8-to-1 Boolean function.

\subsection{Methodology}

First, we describe how to decompose the AES S-box such that the resulting 8-to-1 function is limited to a cubic degree. Next, we illustrate the resulting circuit. Finally, we explain our sharing method.

Using Rotational Symmetry. As noted in [Mor16, NNR18, WM18], the inversion in $\mathrm{GF}\left(2^{8}\right)$ has an algebraic degree of 7 but can be decomposed into two cubic bijections:

$$
x^{-1}=x^{254}=\left(x^{26}\right)^{49}
$$

Since masking with $d+1$ shares for a function with degree $t$ requires at least $(d+1)^{t}$ output shares $\left[\mathrm{RBN}^{+} 15\right]$, we choose to mask the cubic bijections $x^{26}$ and $x^{49}$ instead of realizing $x^{-1}$ in one step. Moreover, since both components of the decomposition are power maps themselves, they can both be implemented using the rotation symmetry approach. Using the same method as before, we can thus find two Boolean functions $G^{*}$ and $F^{*}$ such that $G^{*}(\phi(x))=\phi\left(x^{26}\right)_{0}$ and $F^{*}(\phi(x))=\phi\left(x^{49}\right)_{0}$.

S-box Structure. We illustrate the structure of the decomposed shared S-box in Figure 6. Our purpose is to reuse as much hardware as possible to minimize the utilized FPGA resources. As before, a (shared) byte enters the circuit bit-serially via the input $\boldsymbol{x}_{\boldsymbol{i}}$ and is saved to the upper shift register R1. Each byte share is then transformed to a normal basis representation using the affine mapping $\mathrm{p} 2 \mathrm{n}$. By rotation of $\mathrm{R} 1$, the power map $x^{26}$ is calculated bit by bit using a shared implementation of Boolean function $G^{*}$. The result is shifted bit-wise into the lower register R2 and when completed, the byte is written back into the upper register in parallel. There, it is rotated to calculate the power map $x^{49}$ through shared Boolean function $\boldsymbol{F}^{*}$. When all eight 2 -share bits have been calculated and 
shifted into the lower register, the resulting shares go through the final affine transform, which transforms back into polynomial basis and applies the AES affine function (n2p). The S-box output shares can be obtained bit by bit on wire $\boldsymbol{y}_{\boldsymbol{i}}$.

The block $\boldsymbol{G}^{*} / \boldsymbol{F}^{*}$ can compute either shared Boolean function $\boldsymbol{G}^{*}$ (corresponding to power map $x^{26}$ ) or Boolean function $\boldsymbol{F}^{*}$ (corresponding to power map $x^{49}$ ). Its functionality is determined by a control selection bit.

Masking Cubic Boolean Functions with $\boldsymbol{d}+\mathbf{1}$ shares. Each cubic monomial $a b c$ can be trivially masked with $d+1$ input shares and $(d+1)^{3}$ output shares (one for each crossproduct). For example, a first-order sharing (i.e. $d=1$ ) of $z=a b c$ is given in (1). We designate three domains: one for variable $a$, another for variable $b$ and the third one for variable $c$. In order to not violate the non-completeness, each output share $z_{i}$ contains exactly one share of each domain.

$$
\begin{array}{llll}
z_{0}=a_{0} b_{0} c_{0}, & z_{1}=a_{0} b_{0} c_{1}, & z_{2}=a_{0} b_{1} c_{0}, & z_{3}=a_{0} b_{1} c_{1}, \\
z_{4}=a_{1} b_{0} c_{0}, & z_{5}=a_{1} b_{0} c_{1}, & z_{6}=a_{1} b_{1} c_{0}, & z_{7}=a_{1} b_{1} c_{1}
\end{array}
$$

The result can be compressed back into $d+1$ shares after a refreshing and register stage. Our refreshing strategy resembles that of Domain Oriented Masking [GMK16] in such a way that we apply the same bit of fresh randomness to cross-share terms and do not re-mask inner-share terms:

$$
\begin{aligned}
& z_{0}^{\prime}=\left[z_{0}\right]_{\text {reg }} \oplus\left[z_{1} \oplus r_{0}\right]_{\text {reg }} \oplus\left[z_{2} \oplus r_{1}\right]_{\text {reg }} \oplus\left[z_{3} \oplus r_{2}\right]_{\text {reg }} \\
& z_{1}^{\prime}=\left[z_{4} \oplus r_{2}\right]_{\text {reg }} \oplus\left[z_{5} \oplus r_{1}\right]_{\text {reg }} \oplus\left[z_{6} \oplus r_{0}\right]_{\text {reg }} \oplus\left[z_{7}\right]_{\text {reg }}
\end{aligned}
$$

Note that every term after refreshing e.g. $z_{0}$ or $z_{1} \oplus r_{0}$, is stored in a dedicated register before going to the XOR chain which produces $z_{0}^{\prime}$ and $z_{1}^{\prime}$.

The most basic way to mask a more general cubic function is thus to expand each monomial into eight shares. However, this is wildly inefficient for a Boolean function which can have as many as 20 monomials (in our case). On the other hand, it is impossible to keep certain monomials together without violating non-completeness. Consider for example the function $z=a b c \oplus a b d \oplus a b e \oplus a d e \oplus b d e \oplus c d e$. From the first three monomials, we can appoint variable $a$ to domain 1 , variable $b$ to domain 2 and variables $c, d, e$ to domain 3. However, this designation is inconsistent with the three following monomials, e.g. ade, which would require $d$ and $e$ to be in different domains. It is impossible to create a non-complete sharing of this cubic function with $d+1$ input shares and $(d+1)^{3}$ output shares. Instead, we can split the function into two parts: $a b c \oplus a b d \oplus a b e$ and ade $\oplus$ bde $\oplus c d e$, create independent $d+1$ sharings for each and recombine them afterwards.

Our Heuristic. We developed a heuristic to determine efficient maskings with $d+1$ shares for any cubic Boolean function starting from its unshared algebraic normal form (ANF). Our heuristic is steered by two goals. Firstly, we would like to minimize the number of parts the ANF should be split into in order to achieve non-complete sharings. This is equivalent to limiting the expansion of the number of shares and thus minimizing both the required amount of fresh randomness and the number of registers. Secondly, in order to optimize for a LUT-based implementation, we want to minimize the number of input variables that each function part depends on. Recall that a function of 6 variables fits into 1 LUT and that each additional dependency results in a duplication of the number of LUTs.

Given a cubic ANF, we represent each of its monomials as an ordered triple. We explore different worlds, where a world is defined by the appointment of each input variable to one of three domains $(1,2,3)$. In the above example, we defined the world as $(a: 1, b: 2, c: 3, d: 3, e: 3)$. For functions of algebraic degree $t$ and $n$ inputs, an initial 
preprocessing step iterates through all $t^{n}$ possible worlds and determine which monomials are assignable to it. During this process we eliminate redundant worlds (i.e. with an identical list of assignable monomials) and the worlds without assignable monomials of degree $t$. The next step is function specific: we assign all monomials in the ANF to each consistent world. We first attempt to find one world that can hold all the monomials of the ANF. This is not always possible as the above example illustrates. If this first attempt is unsuccessful, we try to find a split of the ANF. A split is a set of worlds that jointly hold all monomials in the ANF of the Boolean function. In this search, we give preference to splits with a minimum number of worlds, in which each function part depends on a minimum number of variables.

We perform the above described search for all possible normal bases. We note that our search is heuristic and we do not claim optimality.

Implementation Details. We encode worlds and ANFs which are dependent on 8 inputs as a bitvector with 256 entries. An entry in the bitvector at position $m \in \mathrm{GF}\left(2^{8}\right)$ corresponds to one 8-bit monomial $x_{0}^{m_{0}} x_{1}^{m_{1}} \ldots x_{7}^{m_{7}}$ and prescribes whether this monomial is present in the ANF. Recall the ANF of an 8-bit Boolean function $F$ :

$$
F(x)=\bigoplus_{m \in G F\left(2^{8}\right)} a_{m} x_{0}^{m_{0}} x_{1}^{m_{1}} \ldots x_{7}^{m_{7}}
$$

We then define

$$
\operatorname{rep}(F)=\sum_{m} a_{m} 2^{m} \quad \text { and } \quad \operatorname{rep}(w)=\sum_{m} c_{m}^{w} 2^{m}
$$

where $c_{m}^{w}=1$ if monomial $m$ is compatible with world $w$. Consider for example a possible two-split of worlds $\left(w_{1}, w_{2}\right)$. We can determine whether it is a two-split for a Boolean function $F$ by representing both the ANF of $F$ and the list of assignable monomials to each of the worlds as a 256 -bit vector, e.g.

$$
\operatorname{rep}\left(x_{0} x_{2} x_{4} \oplus x_{1} x_{5}\right)=\left(2^{2^{0}+2^{2}+2^{4}}\right) \mid\left(2^{2^{1}+2^{5}}\right)=0 \times 400200000
$$

Now, $\left(w_{1}, w_{2}\right)$ is a two-split for $F$ if the following condition holds:

$$
\operatorname{rep}\left(w_{1}\right)\left|\operatorname{rep}\left(w_{2}\right)\right| \operatorname{rep}(F)=\operatorname{rep}\left(w_{1}\right) \mid \operatorname{rep}\left(w_{2}\right)
$$

where | refers to the Boolean OR-operation. The condition evaluates to true whenever all monomials of the ANF of $F$ are also assignable monomials in at least one of the worlds $w_{1}$ or $w_{2}$.
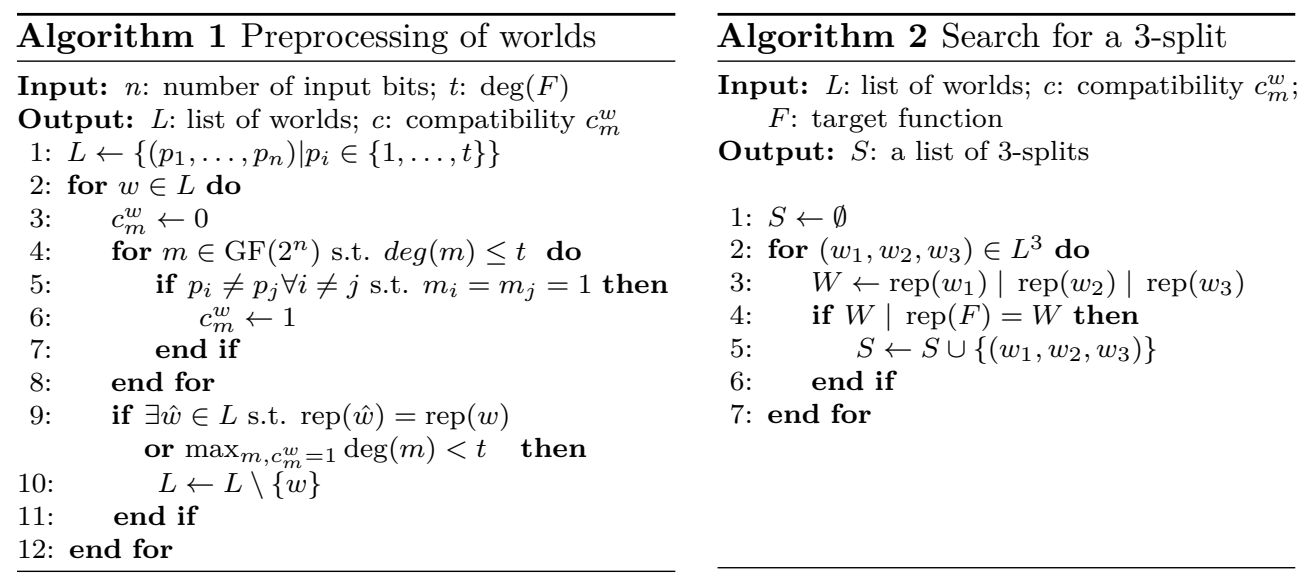
The preprocessing step is illustrated in Algorithm 1 and creates a list of worlds $L$. The list initially contains all $t^{n}$ worlds, i.e. all assignments of $n$ variables $x_{i}$ to one of $t$ domains/positions $p_{i}(1)$. We iterate over $L(2)$. For each monomial $m$ up to the target degree $t(4)$, we check whether it is assignable to the world $w$, i.e. whether for any two variables in the monomial $m$ they do not have the same position in the world $w$ (5). After all assignable monomials for one world have been determined, we check for a duplicate another world with an identical list of assignable monomials - and eliminate. We also check whether the world $w$ contains monomials of the target degree $t$ and otherwise discard it $(9,10)$. The runtime of the entire preprocessing step is bounded by $\mathcal{O}\left(2^{n} \cdot t^{n}\right)$.

Algorithm 2 demonstrates the search for a 3 -split of worlds. Its run-time is $|L|^{3}=\mathcal{O}\left(t^{3 n}\right)$. Using this algorithm, we search through all possible normal bases for splits of the Boolean functions $G^{*}$ and $G^{*} \oplus F^{*}$. For both, the minimum number of worlds in a split we could find is three and the number of input variables for each function part in the 3 -split is seven. Since our methodology is heuristic, we do not claim that a two-split of worlds does not exist for our functions. However, it would probably result in a dependency of more than seven variables, which is less optimal for FPGA implementations. When we apply the algorithm for $F^{*}$, we cannot find a 3-split of worlds where each part depends on at most seven variables. Since we have to implement $G^{*}$ anyway, we can realize the function $F^{*}$ as the XOR of $G^{*}$ and $G^{*} \oplus F^{*}$. Our result reduces the number of occupied LUTs to $50 \%$ compared to a case with dependency on all eight variables. These results were found for a normal basis with $\beta=205$. For the exact equations we refer to Appendix C. Note that the positions of the variables $x_{i}$ in each monomial indicate which domain it belongs to.

\subsection{AES Implementation}

Since our fully bit-serialized design (cf. Table 1; row 4) occupies the smallest area in LUTs and exhibits a lower latency than the byte-serial with bit-serial S-box design based on [SG16] (cf. Table 1; rows 3), we choose to mask this design rather than the byte-serialized architecture.

$\boldsymbol{G}^{*} / \boldsymbol{F}^{*}$. Figure 5 shows the masking of the non-linear block $G^{*} / F^{*}$ in more detail. A control bit sel chooses whether this block computes $G^{*}$ or $\boldsymbol{F}^{*}$. We achieve this by implementing on one hand $G^{*}$ and on the other hand $G^{*} \oplus \boldsymbol{F}^{*}$. The selection bit determines whether the output of the block is the output of only $G^{*}$ or the XOR of both. We split each function $G^{*}$ and $F^{*} \oplus G^{*}$ into three parts $\left[G^{A}, G^{B}, G^{C}\right]$ and $\left[(F \oplus G)^{A},(F \oplus G)^{B},(F \oplus G)^{C}\right]$ using the heuristic described in Section 5.1 and share them individually according to Equation (1) and Equation (2). This means that each part creates eight output shares. The key advantage of our sharing method is that all parts depend on only seven input variables, meaning that each of their output shares can be computed using only two LUTs without mask refreshing or four LUTs (an entire slice) with refreshing. A dependency on all eight inputs would have doubled the resource requirements. As stated before, we only refresh the cross-domain shares. This yields two output shares $\left(z_{0}\right.$ and $\left.z_{7}\right)$ to depend on only seven variables ( $\leq 2$ LUTs) and six output shares to depend on eight variables ( $\leq 4 \mathrm{LUTS})$. Hence, each of the building blocks in the left side of Figure 5 consumes at most 28 LUTs. The resulting $6 \times 8$ output shares are stored in a register to prevent the propagation of glitches. Subsequently, a tree of LUTs does the compression of the shares to $d+1=2$ shares as in Equation (2). Each of the eight LUTs after the register implements a simple XOR of 6 variables. The sel input of the last LUTs determines whether $\boldsymbol{G}^{*}$ or $\left(\boldsymbol{G}^{*} \oplus\left(\boldsymbol{F}^{*} \oplus \boldsymbol{G}^{*}\right)\right)=\boldsymbol{F}^{*}$ is computed. In total, the entire circuit of $\boldsymbol{G}^{*} / \boldsymbol{F}^{*}$ occupies 48 registers and 144 LUTs and exhibits a latency of one clock cycle (due to the compression). 


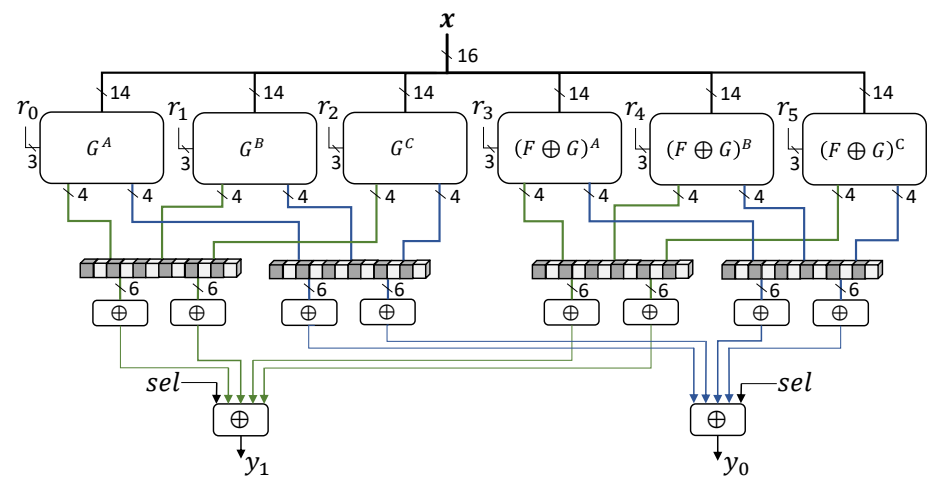

Figure 5: Illustration of the masked realization of the functions $F^{*} / G^{*}$.

Masked S-box. The masked S-box (Figure 6) has a latency of 26 cycles. In clock cycles 1 to 8 , input $\boldsymbol{x}$ is shifted bit-serially into the upper register R1. In cycle 8 , we also apply the affine transform $\mathrm{p} 2 \mathrm{n}$. The evaluation of $G^{*}$ takes one clock cycle because of the register stage between expansion and compression of shares. We use the block as a pipeline, so the upper register R1 rotates continuously in clock cycles 9 to 16 , feeding its content to $\boldsymbol{G}^{*}$ and the results are shifted bit-serially into R2 in clock cycles 10 to 17 . The 7 most significant bits (in 2 shares) of the lower register R2 and the result of the last $G^{*}$ computation are written to the upper register R1 in cycle 17 as well. Then, register R1 rotates again in cycles 18 to 25 and the results of $\boldsymbol{F}^{*}$ are shifted into R2 in clock cycles 19 to 26 . The final affine transform is done in cycle 26 . Result $\boldsymbol{y}$ can then be taken out bit-serially in 8 cycles, but this can be done in parallel with the loading of the next S-box input $\boldsymbol{x}$ into R1.

Vulnerability Potential. When $\mathrm{R} 1$ rotates, the input of $\boldsymbol{F}^{*} / \boldsymbol{G}^{*}$ instantly changes, and this may result in first-order leakage. As an example, consider $x_{1} x_{2} x_{6}$ as one of the terms in the ANF of $G^{B}$ (see Appendix C). Let us denote the value of $\left(x_{1}, x_{2}, x_{3}, x_{6}, x_{7}\right)$ at one clock cycle by $(a, b, c, d, e)$. Based on Equation (1), one of the eight terms in a 2-share realization is $z_{2}=a_{0} b_{1} d_{0}$. In the next clock cycle, register $\mathrm{R} 1$ rotates and $\left(x_{1}, x_{2}, x_{6}\right)$ have the values $(b, c, e)$, hence the same circuit evaluates $z_{2}=b_{0} c_{1} e_{0}$. This means that such a piece of circuit observes $b_{1}$ in one clock cycle, and $b_{0}$ in the next clock cycle. Hence, during the transition (positive edge of the clock) the leakage of the circuit can depend on both shares $b_{0}$ and $b_{1}$, hence breaking the non-completeness and inducing first-order leakage.

In order to avoid this issue, we pre-charge the input of $\boldsymbol{F}^{*} / G^{*}$ before every shift in register R1. To this end, we employ an extra register at $\boldsymbol{F}^{*} / \boldsymbol{G}^{*}$ 's input (see Figure 6), which is triggered at the negative edge of the clock, and reset (clear asynchronously) when clock is high. During the first half of the clock cycle (when clock is high) this pre-charge register clears the input of $\boldsymbol{F}^{*} / G^{*}$. Once the clock changes to low, the value in R1 (already shifted) is stored in the register, hence given to $F^{*} / G^{*}$. At the next positive edge of the clock, R1 shifts and at the same time the pre-charge register is cleared, thereby pre-charging the $\boldsymbol{F}^{*} / G^{*}$ input. This construction prevents any race between R1 being shifted and the pre-charge register being cleared. Even if R1 is shifted earlier (since its clock should have low skew) this transition does not pass through the pre-charge register, and $\boldsymbol{F}^{*} / \boldsymbol{G}^{*}$ 's input stays unchanged.

As a disadvantage, this construction can theoretically halve the maximum clock frequency. However, we have observed that $\boldsymbol{F}^{*} / G^{*}$ is not involved in the critical path of the circuit realizing the full AES encryption. Hence, the maximum clock frequency is not very much affected, and can even be maintained if the duty cycle of the clock is properly adjusted.

With respect to implementation, the $\boldsymbol{F}^{*} / \boldsymbol{G}^{*}$ block requires 144 LUTs and 48 flip-flops. 


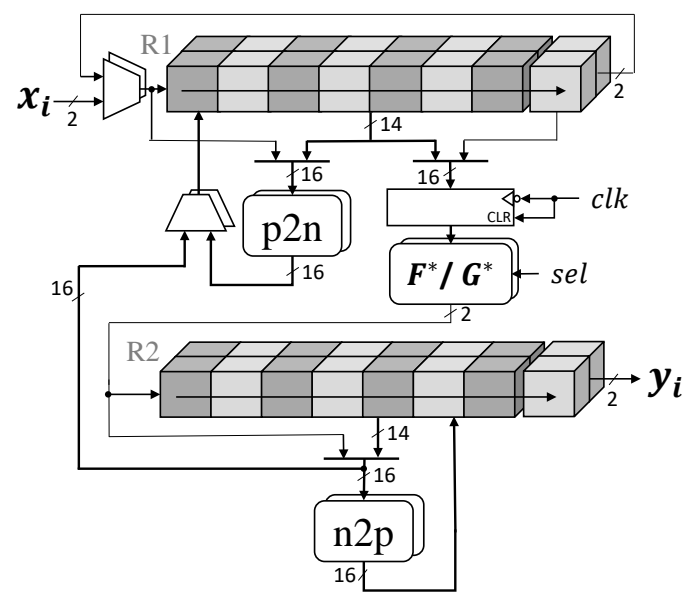

Figure 6: Illustration of the first-order secure AES S-box based on rotational symmetry when decomposed into $x^{26}$ and $x^{49}$.

In addition, for each share we need 7 LUTs for both p2n and n2p, 1 LUT for the addition of the round key and 4 LUTs for the multiplexer that chooses the parallel input to R1. Each share also requires two 8-bit registers (R1 and R2) as well as one 8-bit register for the precharging of the $\boldsymbol{F}^{*} / \boldsymbol{G}^{*}$ input. Therefore, our masked S-box can be implemented with $(144+2 \times(7+7+4+1))=182$ LUTs and $(48+2 \times(8+8+8))=96$ flip-flops. Further, the S-box has a fresh randomness cost of $6 \times 3=18$ bits per $\boldsymbol{F}^{*} / \boldsymbol{G}^{*}$ evaluation, i.e. 18 bits per clock cycle. Each group of 3 bits is used in one part of the shared Boolean function as in Equation (2) (see Figure 5 with $r_{i} \in \mathrm{GF}(2)^{3}$ ).

Full-AES. We integrate the S-box into the same bit-serial AES design as used in Section 4. The state and key array and linear components of the AES cipher (MixColumns, AddRoundKey and ShiftRows) have simply been duplicated for each share separately. This results in occupying $23 \times 2=46$ LUTs and $4 \times 2=8$ registers. The latency of ShiftRows and MixColumns stays the same as for an unmasked design. When plugging in the masked S-box, we also need to adapt our control logic since the S-box latency has changed and we require an extra control signal to select $\boldsymbol{G}^{*}$ or $\boldsymbol{F}^{*}$. This new control unit uses 31 LUTs and 20 flip-flops. The design has a latency of 676 cycles per round with a shorter last round of 640 cycles. In total, with 128 cycles of loading, one encryption takes 6852 cycles. The total footprint of our masked AES (post-map) is 124 flip-flops and 293 LUTs when the key schedule is masked and 283 LUTs when it is not.

Results. It is difficult to compare these results to state-of-the-art masked AES implementations $\left[\mathrm{BGN}^{+} 15, \mathrm{CRB}^{+} 16, \mathrm{GMK} 17, \mathrm{UHA} 17 \mathrm{~b}\right]$ since they target an ASIC platform. We can let Xilinx map these designs to Spartan-6 resources, but unlike our design, they have not been optimized specifically for this purpose. In Table 2, we do this first for various masked S-box implementations. The results from other works are obtained by synthesis, translate and map using Xilinx default settings apart from the KEEP HIERARCHY constraint which is turned on to prohibit optimization across shares. We stress that no optimization for FPGA has been done for these designs. When comparing these results to the ASIC numbers reported in the original works, the stark contrast between the worlds of ASICs and FPGAs is clearly confirmed. Moreover, the FPGA footprint is strongly influenced by the coding style of the creators (e.g. extent of hierarchy use, clock gating vs. clock enabling, ...), which is obviously different for each of the designs.

We should emphasize that all the considered designs are expected to provide only 
first-order security with minimum number of shares for the state and key arrays. The random bits, which we report in Table 2, are corresponding to the number of fresh random bits required at each clock cycle. Since the other designs have a pipelined S-box, the number of required fresh masks per clock cycle is the same as those required for every S-box evaluation. However, since in our design the pipelining is done only for the $G^{*} / F^{*}$ block, the number of required fresh masks per S-box invocation is different. In contrast, $G^{*} / F^{*}$ is the only block which requires fresh masks, i.e. 18 bits per clock cycle.

We further report the same performance figures for the corresponding full AES encryption-only implementations in Table $3^{6}$. Note that for all these designs, both the state and key arrays are shared.

Table 2: Comparison of first-order secure AES S-boxes, mapped for Spartan-6.

\begin{tabular}{lcccc}
\hline Design & \# LUTs & \# FFs & \# Slices & \# Random bits \\
\hline Bilgin et al. $\left[\mathrm{BGN}^{+} 15\right]$ & 361 & 92 & 177 & 32 \\
Gross et al. $\left[\mathrm{GMK}^{+}\right]$ & 327 & 208 & 242 & 18 \\
Cnudde et al. $\left[\mathrm{CRB}^{+}\right.$16] & 340 & 144 & 283 & 54 \\
Ueno et al. $[\mathrm{UHA} 17 \mathrm{~b}]$ & 302 & 96 & 218 & 64 \\
\hline This work & 182 & 96 & 95 & 18 \\
\hline
\end{tabular}

Table 3: Comparison of first-order secure AES implementations, mapped for Spartan-6.

\begin{tabular}{lccccc}
\hline Design & \# LUTs & \# FFs & \# Slices & \# Clocks & $\mathbf{f}_{\max }$ \\
\hline Bilgin et al. (nimble) $\left[\mathrm{BGN}^{+} 15\right]$ & 1198 & 611 & 475 & 246 & $127 \mathrm{MHz}$ \\
Gross et al. $[\mathrm{GMK17}]$ & 595 & 734 & 366 & 246 & $103 \mathrm{MHz}$ \\
Cnudde et al. $\left[\mathrm{CRB}^{+} 16\right]$ & 1191 & 642 & 553 & 276 & $181 \mathrm{MHz}$ \\
\hline This work & 293 & 124 & 162 & 6852 & $103 \mathrm{MHz}$ \\
\hline
\end{tabular}

\subsection{SCA Evaluation}

VerMI. As a first evaluation step, we use the VerMI tool [ANR17], which was specifically created to verify the security of masked implementations in the presence of glitches. This tool can be used directly on our VHDL code and confirms the non-completeness and uniformity of the $\boldsymbol{F}^{*} / G^{*}$ block.

Measurement Setup. For practical evaluations, we implement our full AES encryption design on the target Spartan-6 FPGA of the SAKURA-G platform [sak], a commonly known and employed board for SCA evaluations. By means of a digital oscilloscope at a sampling rate of $625 \mathrm{MS} / \mathrm{s}$, we measure the power consumption of the target FPGA, which is clocked at a frequency of $6 \mathrm{MHz}$, through the dedicated on-board $\mathrm{AC}$ amplifier. Due to the very low power consumption of our design (particularly since the state and key arrays are stored in shift register LUTs), we additionally employ an AC amplifier ${ }^{7}$ with $10 \mathrm{~dB}$ gain. During the measurements, the masked AES core receives the shared plaintext and the shared key and sends back the shared ciphertext.

Each of the required 18-bit fresh masks are provided by a dedicated 31-bit LFSR with the feedback polynomial $x^{31}+x^{28}+1$. Such an LFSR has a maximum cycle $2^{31}-1$ with only two taps [WM12], hence should suffice for more than 2 billion measurements. Each LFSR is implemented by means of only 3 LUTs, of which two are employed as shift register and the last one to make the feedback signal, i.e. the entire fresh mask generation is realized in $18 \times 3=54$ LUTs. We arbitrarily initialize the LFSRs (not null) right after the

\footnotetext{
${ }^{6}$ We do not have access to the design of the full AES implementation of [UHA17b].
}

${ }^{7}$ ZFL-1000LN+ from Mini-Circuits 
FPGA power-up. They are supplied with the same clock as the masked AES core, but operate on the negative edge of the clock. This is done to reduce the effect of the LFSR transitions on the SCA measurements associated to the masked AES core $\left[\mathrm{CRB}^{+} 16\right]$.

Evaluation. Most of the related state-of-the-art schemes evaluate the masked design by means of fixed-versus-random t-test [GJJR11, $\mathrm{CDG}^{+} 13$, SM15]. It has recently been shown that such evaluations on masked hardware with only 2 shares can yield misleading results [CEM18]. In other words, when the measurement noise is low, such a t-test may always show detectable leakage independent of the implementation and the underlying masking scheme. Since our design is also prone to this issue due to its very low resource requirements, we conduct attacks instead of such leakage assessment techniques. To this end, in order to relax the necessity of having a detailed and accurate power consumption model, we decide to perform Moments-Correlating DPA [MS16] (MC-DPA) which is a more robust and theoretically more accurate form of Correlation-Enhanced Collision Attack [MME10]. In short, we perform first- and second-order collision Moment-Correlation DPA attacks by considering the leakage of one S-box evaluation as the model and thereby performing the attack on another S-box evaluation. It is noteworthy that such linear collision attacks recover the linear difference between the associated keys [Bog08].

PRNG OFF. We first turn off the LFSR PRNG (for the fresh masks) as well as the initial masking of the plaintext and key to emulate an unprotected implementation. The sample trace shown in Figure 7a covers eight S-box evaluations of the first encryption round (indeed of the first two state rows). We also present the signal-to-noise ratio (SNR) curves estimated based on the value of the plaintext bytes in Figure $7 \mathrm{~b}$. To this end, we follow the procedure explained in [MOP07]. The SNR curves show a clear dependency on the plaintext bytes, and hence the S-box inputs. Using 10000 traces and considering the leakage of the second S-box evaluation (of state byte no. 4) as the model, we conduct a first-order MC-DPA on the third S-box (of state byte no. 8), which yields the correlation curves shown in Figure 7c. The results indicate that very few traces are required to correctly identify the difference between the corresponding key bytes. We further repeat the same experiment for two other cases: (a) LFSR PRNG on and initial masking off, (b) LFSR PRNG off and initial masking on. For both cases we again observe clearly-distinguishable SNR curves (although with lower amplitude, i.e. 0.02 compared to 13 in Figure 7b). The same MC-DPA attacks also successfully recover the correct key difference using at most 100000 traces.

PRNG ON. When both the LFSR PRNG and initial masking are active, we collect 10000000 traces, each covering only the above-selected two S-box evaluations ${ }^{8}$. Following the same scenario as in the case PRNG off, we perform both first-order and second-order MC-DPA attacks. The corresponding results are shown in Figure 8. It is noticeable that although the first-order leakage cannot be exploited, the second-order attack succeeds with very low number of,e.g. 10000 traces. This is due to two facts: (a) masking with minimum number of two shares has in general a strong vulnerability to second-order attacks [CFE16], (b) higher-order attacks are sensitive to the noise level [PRB09] and our design (due to its extremely low resource utilization) has a very low switching noise particularly when the masked S-box is evaluated the entire circuit stops till the termination of the S-box. Hence, the S-box is the sole source of leakage at that time. Further, our utilized LFSR PRNG (again using shift register LUTs) does not add a remarkable amount of noise to the measurements. In such cases, adding noise modules would surely help to harden higher-order attacks. As an example we refer to [EGMP17], where the design of such a noise generator on the same FPGA type is given.

\footnotetext{
${ }^{8}$ Due to the high latency of the entire encryption, the measurement process is relatively slow. We also have to cover at least two S-box evaluations (for collision MC-DPA) leading to long power traces. This limited our analysis with respect to the number of collected traces.
} 


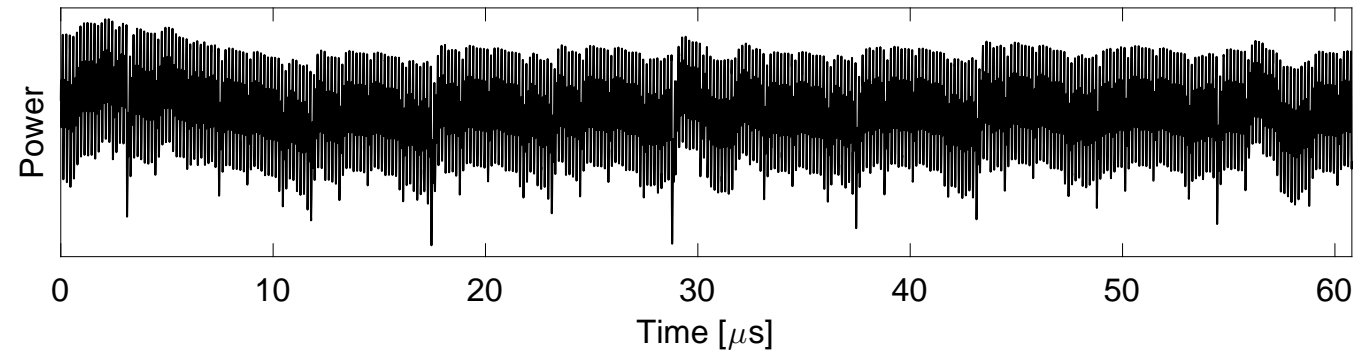

(a) sample trace

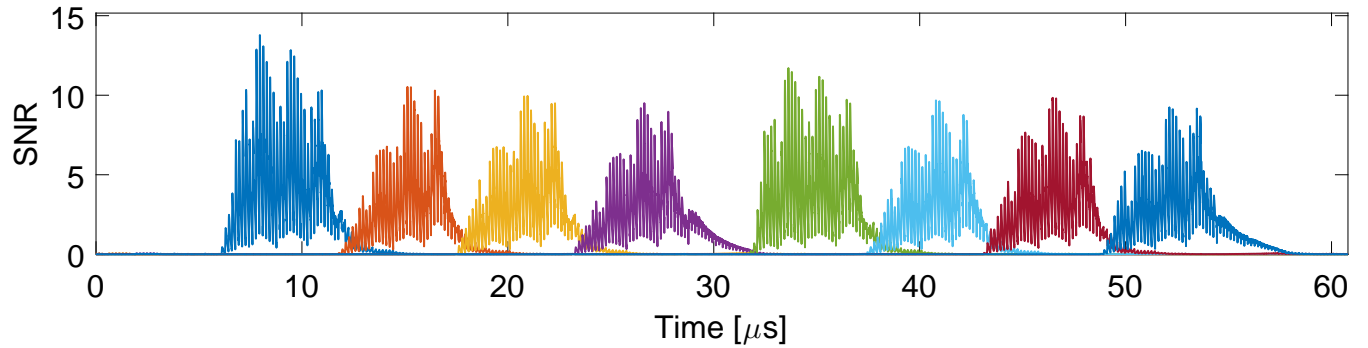

(b) SNR

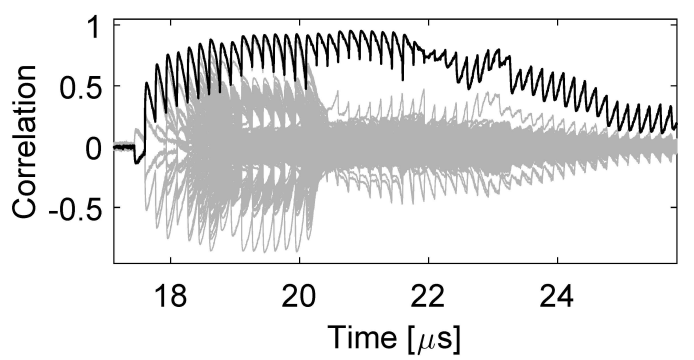

(c) MC-DPA, first-order

Figure 7: PRNG and initial masking disabled, 10000 traces, (a) sample trace, (b) SNR curves based on 8 plaintext bytes with the order from left to right: byte no. $0,4,8,12,1$, 5, 9, 13, (c) first-order Moments-Correlating DPA result targeting S-box no. 8 with model S-box no. 4, the black curve belonging to the correct key difference.

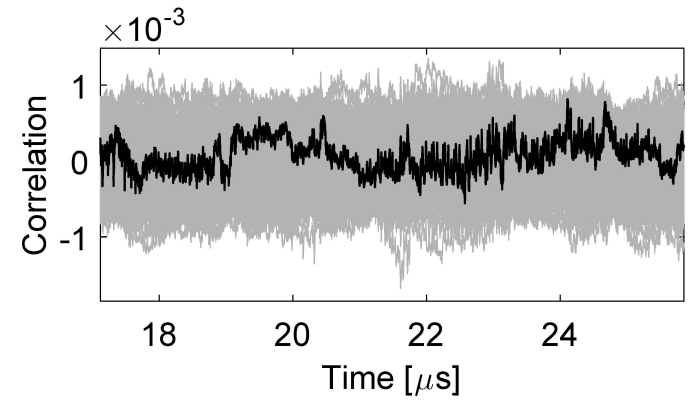

(a) MC-DPA, first-order

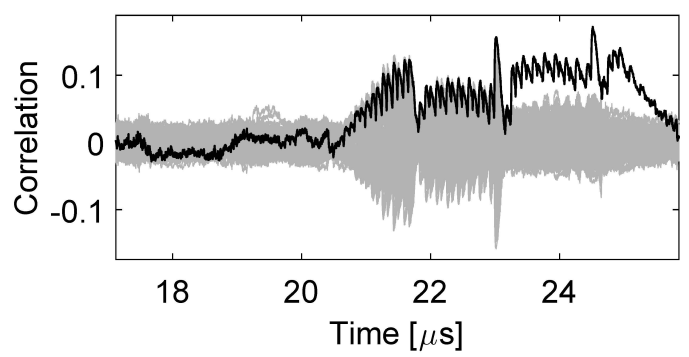

(b) MC-DPA, second-order

Figure 8: PRNG and initial masking enabled, Moments-Correlating DPA result targeting S-box no. 8 with model S-box no. 4, (a) first-order with 10000000 traces, (b) second-order with 10000 traces. 


\section{Conclusion}

Our contribution is manifold. First, we made several FPGA-specific AES implementations which compromise between the latency and area requirements. We improved the latency of the formerly smallest known AES on Xilinx FPGAs [SG16]. Furthermore, we achieved a new size record by replacing its S-box with our bit-serial rotational design fitting into only 17 slices, while the former record by Sasdrich et al. [SG16] requires 21 slices - a 19\% size reduction. This can be fully attributed to cutting the size of the S-box by half from 8 slices to 4 .

Second, with respect to masking as an SCA countermeasure, we developed an effective heuristic to find sharings of Boolean functions with $d+1$ shares by determining variable positions in monomials and splitting the Boolean function ANF into multiple components. This has the advantage of reducing the number of variables the function depends upon, thereby yielding an area reduction on LUT-based architectures such as FPGAs.

Third, we applied our heuristic and demonstrated that masking with $d+1$ shares is easily applied to our AES S-box construction. We exploit the rotational symmetry of a cubic decomposition of the inversion in $\operatorname{GF}\left(2^{8}\right)$ which further limits the area overhead. Our first-order secure AES S-box requires only 182 LUTS, while the masked AES encryption requires 293 LUTs - another area record on FPGAs. However, we should emphasize that such low area footprints come at the cost of high latency. More precisely, our designs are suitable for applications with no high throughput needs. To promote further research as well as for comparison purposes, the HDL code of our implementations is publicly available through the authors' webpage.

\section{Acknowledgments}

The work described in this paper has been supported in part by the German Federal Ministry of Education and Research BMBF (grant nr. 16KIS0666 SysKit_HW). Lauren De Meyer is funded by a PhD fellowship of the Fund for Scientific Research - Flanders (FWO).

\section{References}

[ANR17] Victor Arribas, Svetla Nikova, and Vincent Rijmen. VerMI: Verification tool for masked implementations. IACR Cryptology ePrint Archive, 2017:1227, 2017.

[BB16] Tim Beyne and Begül Bilgin. Uniform first-order threshold implementations. In Roberto Avanzi and Howard M. Heys, editors, Selected Areas in Cryptography SAC 2016 - 23rd International Conference, St. John's, NL, Canada, August 1012, 2016, Revised Selected Papers, volume 10532 of Lecture Notes in Computer Science, pages 79-98. Springer, 2016.

$\left[\mathrm{BDF}^{+} 17\right]$ Gilles Barthe, François Dupressoir, Sebastian Faust, Benjamin Grégoire, François-Xavier Standaert, and Pierre-Yves Strub. Parallel implementations of masking schemes and the bounded moment leakage model. In Jean-Sébastien Coron and Jesper Buus Nielsen, editors, Advances in Cryptology - EUROCRYPT 2017 - 36th Annual International Conference on the Theory and Applications of Cryptographic Techniques, Paris, France, April 30 - May 4, 2017, Proceedings, Part I, volume 10210 of Lecture Notes in Computer Science, pages 535-566, 2017. 
[BDGH15] Shivam Bhasin, Jean-Luc Danger, Sylvain Guilley, and Wei He. Exploiting FPGA block memories for protected cryptographic implementations. TRETS, 8(3):16:1-16:16, 2015.

[BGD12] Shivam Bhasin, Sylvain Guilley, and Jean-Luc Danger. From cryptography to hardware: Analyzing embedded xilinx BRAM for cryptographic applications. In 45th Annual IEEE/ACM International Symposium on Microarchitecture, MICRO 2012, Workshops Proceedings, Vancouver, BC, Canada, December 1-5, 2012, pages 1-8. IEEE Computer Society, 2012.

$\left[\mathrm{BGN}^{+} 15\right]$ Begül Bilgin, Benedikt Gierlichs, Svetla Nikova, Ventzislav Nikov, and Vincent Rijmen. Trade-offs for threshold implementations illustrated on AES. IEEE Trans. on CAD of Integrated Circuits and Systems, 34(7):1188-1200, 2015.

$\left[\mathrm{BGS}^{+} 11\right]$ Shivam Bhasin, Sylvain Guilley, Youssef Souissi, Tarik Graba, and JeanLuc Danger. Efficient dual-rail implementations in FPGA using block rams. In Peter M. Athanas, Jürgen Becker, and René Cumplido, editors, 2011 International Conference on Reconfigurable Computing and FPGAs, ReConFig 2011, Cancun, Mexico, November 30 - December 2, 2011, pages 261-267. IEEE Computer Society, 2011.

[BMP13] Joan Boyar, Philip Matthews, and René Peralta. Logic minimization techniques with applications to cryptology. J. Cryptology, 26(2):280-312, 2013.

$\left[\mathrm{BNN}^{+}{ }^{12}\right]$ Begül Bilgin, Svetla Nikova, Ventzislav Nikov, Vincent Rijmen, and Georg Stütz. Threshold implementations of all 3x3 and 4x4 s-boxes. In Emmanuel Prouff and Patrick Schaumont, editors, Cryptographic Hardware and Embedded Systems - CHES 2012 - 14th International Workshop, Leuven, Belgium, September 9-12, 2012. Proceedings, volume 7428 of Lecture Notes in Computer Science, pages 76-91. Springer, 2012.

[Bog08] Andrey Bogdanov. Multiple-differential side-channel collision attacks on AES. In Elisabeth Oswald and Pankaj Rohatgi, editors, Cryptographic Hardware and Embedded Systems - CHES 2008, 10th International Workshop, Washington, D.C., USA, August 10-13, 2008. Proceedings, volume 5154 of Lecture Notes in Computer Science, pages 30-44. Springer, 2008.

$\left[\mathrm{BSQ}^{+} 08\right]$ Philippe Bulens, François-Xavier Standaert, Jean-Jacques Quisquater, Pascal Pellegrin, and Gaël Rouvroy. Implementation of the AES-128 on Virtex-5 FPGAs. In Serge Vaudenay, editor, Progress in Cryptology - AFRICACRYPT 2008, First International Conference on Cryptology in Africa, Casablanca, Morocco, June 11-14, 2008. Proceedings, volume 5023 of Lecture Notes in Computer Science, pages 16-26. Springer, 2008.

[Can05] David Canright. A very compact s-box for AES. In Rao and Sunar [RS05], pages $441-455$.

[CB08] David Canright and Lejla Batina. A very compact "perfectly masked" S-Box for AES. In Steven M. Bellovin, Rosario Gennaro, Angelos D. Keromytis, and Moti Yung, editors, Applied Cryptography and Network Security, 6th International Conference, ACNS 2008, New York, NY, USA, June 3-6, 2008. Proceedings, volume 5037 of Lecture Notes in Computer Science, pages 446-459, 2008.

[CB12] Junfeng Chu and Mohammed Benaissa. Low area memory-free FPGA implementation of the AES algorithm. In Dirk Koch, Satnam Singh, and Jim Tørresen, editors, 22nd International Conference on Field Programmable Logic 
and Applications (FPL), Oslo, Norway, August 29-31, 2012, pages 623-626. IEEE, 2012.

$\left[\mathrm{CDG}^{+} 13\right]$ Jeremy Cooper, Elke De Mulder, Gilbert Goodwill, Joshua Jaffe, Gary Kenworthy, and Pankaj Rohatgi. Test Vector Leakage Assessment (TVLA) Methodology in Practice. International Cryptographic Module Conference, 2013.

[CEM18] Thomas De Cnudde, Maik Ender, and Amir Moradi. Hardware masking, revisited. IACR Transactions on Cryptographic Hardware and Embedded Systems, 2018(2), 2018. to appear.

[CFE16] Cong Chen, Mohammad Farmani, and Thomas Eisenbarth. A tale of two shares: Why two-share threshold implementation seems worthwhile - and why it is not. In Jung Hee Cheon and Tsuyoshi Takagi, editors, Advances in Cryptology - ASIACRYPT 2016 - 22nd International Conference on the Theory and Application of Cryptology and Information Security, Hanoi, Vietnam, December 4-8, 2016, Proceedings, Part I, volume 10031 of Lecture Notes in Computer Science, pages 819-843, 2016.

[CG03] Pawel Chodowiec and Kris Gaj. Very compact FPGA implementation of the AES algorithm. In Colin D. Walter, Çetin Kaya Koç, and Christof Paar, editors, Cryptographic Hardware and Embedded Systems - CHES 2003, 5th International Workshop, Cologne, Germany, September 8-10, 2003, Proceedings, volume 2779 of Lecture Notes in Computer Science, pages 319-333. Springer, 2003.

[CJRR99] Suresh Chari, Charanjit S. Jutla, Josyula R. Rao, and Pankaj Rohatgi. Towards sound approaches to counteract power-analysis attacks. In Wiener [Wie99], pages $398-412$.

$\left[\mathrm{CRB}^{+} 16\right]$ Thomas De Cnudde, Oscar Reparaz, Begül Bilgin, Svetla Nikova, Ventzislav Nikov, and Vincent Rijmen. Masking AES with $\mathrm{d}+1$ shares in hardware. In Benedikt Gierlichs and Axel Y. Poschmann, editors, Cryptographic Hardware and Embedded Systems - CHES 2016 - 18th International Conference, Santa Barbara, CA, USA, August 17-19, 2016, Proceedings, volume 9813 of Lecture Notes in Computer Science, pages 194-212. Springer, 2016.

[EGMP17] Maik Ender, Samaneh Ghandali, Amir Moradi, and Christof Paar. The first thorough side-channel hardware trojan. In Tsuyoshi Takagi and Thomas Peyrin, editors, Advances in Cryptology - ASIACRYPT 2017 - 23rd International Conference on the Theory and Applications of Cryptology and Information Security, Hong Kong, China, December 3-7, 2017, Proceedings, Part I, volume 10624 of Lecture Notes in Computer Science, pages 755-780. Springer, 2017.

[GH15] Tim Güneysu and Helena Handschuh, editors. Cryptographic Hardware and Embedded Systems - CHES 2015 - 17th International Workshop, Saint-Malo, France, September 13-16, 2015, Proceedings, volume 9293 of Lecture Notes in Computer Science. Springer, 2015.

[GJJR11] Gilbert Goodwill, Benjamin Jun, Josh Jaffe, and Pankaj Rohatgi. A testing methodology for Side channel resistance validation. In NIST Non-invasive Attack Testing Workshop, 2011.

[GMK16] Hannes Groß, Stefan Mangard, and Thomas Korak. Domain-oriented masking: Compact masked hardware implementations with arbitrary protection order. IACR Cryptology ePrint Archive, 2016:486, 2016. 
[GMK17] Hannes Groß, Stefan Mangard, and Thomas Korak. An efficient side-channel protected AES implementation with arbitrary protection order. In Helena Handschuh, editor, Topics in Cryptology - CT-RSA 2017 - The Cryptographers' Track at the RSA Conference 2017, San Francisco, CA, USA, February 14-17, 2017, Proceedings, volume 10159 of Lecture Notes in Computer Science, pages 95-112. Springer, 2017.

[ISW03] Yuval Ishai, Amit Sahai, and David A. Wagner. Private circuits: Securing hardware against probing attacks. In Dan Boneh, editor, Advances in Cryptology - CRYPTO 2003, 23rd Annual International Cryptology Conference, Santa Barbara, California, USA, August 17-21, 2003, Proceedings, volume 2729 of Lecture Notes in Computer Science, pages 463-481. Springer, 2003.

[JMPS17] Jérémy Jean, Amir Moradi, Thomas Peyrin, and Pascal Sasdrich. Bit-sliding: A generic technique for bit-serial implementations of SPN-based primitives - applications to AES, PRESENT and SKINNY. In Wieland Fischer and Naofumi Homma, editors, Cryptographic Hardware and Embedded Systems CHES 2017 - 19th International Conference, Taipei, Taiwan, September 25-28, 2017, Proceedings, volume 10529 of Lecture Notes in Computer Science, pages 687-707. Springer, 2017.

[KJJ99] Paul C. Kocher, Joshua Jaffe, and Benjamin Jun. Differential power analysis. In Wiener [Wie99], pages 388-397.

[MBPV05] Nele Mentens, Lejla Batina, Bart Preneel, and Ingrid Verbauwhede. A systematic evaluation of compact hardware implementations for the Rijndael S-Box. In Alfred Menezes, editor, Topics in Cryptology - CT-RSA 2005, The Cryptographers' Track at the RSA Conference 2005, San Francisco, CA, USA, February 14-18, 2005, Proceedings, volume 3376 of Lecture Notes in Computer Science, pages 323-333. Springer, 2005.

[MM12] Amir Moradi and Oliver Mischke. Glitch-free implementation of masking in modern fpgas. In 2012 IEEE International Symposium on Hardware-Oriented Security and Trust, HOST 2012, San Francisco, CA, USA, June 3-4, 2012, pages 89-95. IEEE, 2012.

[MME10] Amir Moradi, Oliver Mischke, and Thomas Eisenbarth. Correlation-enhanced power analysis collision attack. In Stefan Mangard and François-Xavier Standaert, editors, Cryptographic Hardware and Embedded Systems, CHES 2010, 12th International Workshop, Santa Barbara, CA, USA, August 17-20, 2010. Proceedings, volume 6225 of Lecture Notes in Computer Science, pages 125-139. Springer, 2010.

[MOP07] Stefan Mangard, Elisabeth Oswald, and Thomas Popp. Power analysis attacks - revealing the secrets of smart cards. Springer, 2007.

[Mor16] Amir Moradi. Advances in Side-channel Security, 2016.

$\left[\mathrm{MPL}^{+} 11\right]$ Amir Moradi, Axel Poschmann, San Ling, Christof Paar, and Huaxiong Wang. Pushing the limits: A very compact and a threshold implementation of AES. In Kenneth G. Paterson, editor, Advances in Cryptology - EUROCRYPT 2011 - 30th Annual International Conference on the Theory and Applications of Cryptographic Techniques, Tallinn, Estonia, May 15-19, 2011. Proceedings, volume 6632 of Lecture Notes in Computer Science, pages 69-88. Springer, 2011 . 
[MPO05] Stefan Mangard, Norbert Pramstaller, and Elisabeth Oswald. Successfully attacking masked AES hardware implementations. In Rao and Sunar [RS05], pages 157-171.

[MS16] Amir Moradi and François-Xavier Standaert. Moments-correlating DPA. In Begül Bilgin, Svetla Nikova, and Vincent Rijmen, editors, Proceedings of the ACM Workshop on Theory of Implementation Security, TIS@CCS 2016 Vienna, Austria, October, 2016, pages 5-15. ACM, 2016.

[MW15] Amir Moradi and Alexander Wild. Assessment of Hiding the Higher-Order Leakages in Hardware - What Are the Achievements Versus Overheads? In Güneysu and Handschuh [GH15], pages 453-474.

$\left[\mathrm{NBD}^{+} 10\right]$ Maxime Nassar, Shivam Bhasin, Jean-Luc Danger, Guillaume Duc, and Sylvain Guilley. BCDL: A high speed balanced DPL for FPGA with global precharge and no early evaluation. In Giovanni De Micheli, Bashir M. Al-Hashimi, Wolfgang Müller, and Enrico Macii, editors, Design, Automation and Test in Europe, DATE 2010, Dresden, Germany, March 8-12, 2010, pages 849-854. IEEE Computer Society, 2010.

[NNR18] Svetla Nikova, Ventzislav Nikov, and Vincent Rijmen. Decomposition of permutations in a finite field. IACR Cryptology ePrint Archive, 2018:103, 2018.

[NRR06] Svetla Nikova, Christian Rechberger, and Vincent Rijmen. Threshold implementations against side-channel attacks and glitches. In Peng Ning, Sihan Qing, and Ninghui Li, editors, Information and Communications Security, 8th International Conference, ICICS 2006, Raleigh, NC, USA, December 4-7, 2006, Proceedings, volume 4307 of Lecture Notes in Computer Science, pages 529-545. Springer, 2006.

[NRS08] Svetla Nikova, Vincent Rijmen, and Martin Schläffer. Using normal bases for compact hardware implementations of the AES s-box. In Rafail Ostrovsky, Roberto De Prisco, and Ivan Visconti, editors, Security and Cryptography for Networks, 6th International Conference, SCN 2008, Amalfi, Italy, September 10-12, 2008. Proceedings, volume 5229 of Lecture Notes in Computer Science, pages 236-245. Springer, 2008.

[NRS11] Svetla Nikova, Vincent Rijmen, and Martin Schläffer. Secure hardware implementation of nonlinear functions in the presence of glitches. J. Cryptology, 24(2):292-321, 2011.

[PRB09] Emmanuel Prouff, Matthieu Rivain, and Régis Bevan. Statistical analysis of second order differential power analysis. IEEE Trans. Computers, 58(6):799$811,2009$.

[RBF08] Vincent Rijmen, Paulo S. L. M. Barreto, and Décio L. Gazzoni Filho. Rotation symmetry in algebraically generated cryptographic substitution tables. Inf. Process. Lett., 106(6):246-250, 2008.

$\left[\mathrm{RBN}^{+} 15\right]$ Oscar Reparaz, Begül Bilgin, Svetla Nikova, Benedikt Gierlichs, and Ingrid Verbauwhede. Consolidating masking schemes. In Rosario Gennaro and Matthew Robshaw, editors, Advances in Cryptology - CRYPTO 2015 - 35th Annual Cryptology Conference, Santa Barbara, CA, USA, August 16-20, 2015, Proceedings, Part I, volume 9215 of Lecture Notes in Computer Science, pages 764-783. Springer, 2015. 
[RS05] Josyula R. Rao and Berk Sunar, editors. Cryptographic Hardware and Embedded Systems - CHES 2005, 7th International Workshop, Edinburgh, UK, August 29 - September 1, 2005, Proceedings, volume 3659 of Lecture Notes in Computer Science. Springer, 2005.

[RWS11] Francesco Regazzoni, Yi Wang, and François-Xavier Standaert. Fpga implementations of the aes masked against power analysis attacks. In Constructive Side-Channel Analysis and Secure Design - 2nd International Workshop, COSADE 2011, Darmstadt, Germany, February 24-25, 2011. Revised Selected Papers, pages 56-66, 2011.

[sak] Side-channel AttacK User Reference Architecture. http://satoh.cs.uec.ac. jp/SAKURA/index.html.

[SG16] Pascal Sasdrich and Tim Güneysu. A grain in the silicon: SCA-protected AES in less than 30 slices. In 27th IEEE International Conference on Applicationspecific Systems, Architectures and Processors, ASAP 2016, London, United Kingdom, July 6-8, 2016, pages 25-32. IEEE Computer Society, 2016.

[SM15] Tobias Schneider and Amir Moradi. Leakage assessment methodology - A clear roadmap for side-channel evaluations. In Güneysu and Handschuh [GH15], pages $495-513$.

[SMMG15] Pascal Sasdrich, Oliver Mischke, Amir Moradi, and Tim Güneysu. Side-channel protection by randomizing look-up tables on reconfigurable hardware - pitfalls of memory primitives. In Stefan Mangard and Axel Y. Poschmann, editors, Constructive Side-Channel Analysis and Secure Design - 6th International Workshop, COSADE 2015, Berlin, Germany, April 13-14, 2015. Revised Selected Papers, volume 9064 of Lecture Notes in Computer Science, pages 95-107. Springer, 2015.

[SMTM01] Akashi Satoh, Sumio Morioka, Kohji Takano, and Seiji Munetoh. A compact rijndael hardware architecture with s-box optimization. In Colin Boyd, editor, Advances in Cryptology - ASIACRYPT 2001, 7th International Conference on the Theory and Application of Cryptology and Information Security, Gold Coast, Australia, December 9-13, 2001, Proceedings, volume 2248 of Lecture Notes in Computer Science, pages 239-254. Springer, 2001.

[Tri03] Elena Trichina. Combinational logic design for AES subbyte transformation on masked data. IACR Cryptology ePrint Archive, 2003:236, 2003.

[UHA17a] Rei Ueno, Naofumi Homma, and Takafumi Aoki. A systematic design of tamperresistant galois-field arithmetic circuits based on threshold implementation with $(\mathrm{d}+1)$ input shares. In 47 th IEEE International Symposium on MultipleValued Logic, ISMVL 2017, Novi Sad, Serbia, May 22-24, 2017, pages 136-141. IEEE Computer Society, 2017.

[UHA17b] Rei Ueno, Naofumi Homma, and Takafumi Aoki. Toward more efficient dparesistant AES hardware architecture based on threshold implementation. In Sylvain Guilley, editor, Constructive Side-Channel Analysis and Secure Design - 8th International Workshop, COSADE 2017, Paris, France, April 13-14, 2017, Revised Selected Papers, volume 10348 of Lecture Notes in Computer Science, pages 50-64. Springer, 2017.

[VRM17] Jo Vliegen, Oscar Reparaz, and Nele Mentens. Maximizing the throughput of threshold-protected AES-GCM implementations on FPGA. In IEEE 2nd 
International Verification and Security Workshop, IVSW 2017, Thessaloniki, Greece, July 3-5, 2017, pages 140-145. IEEE, 2017.

[Wam14] Markus Stefan Wamser. Ultra-small designs for inversion-based S-Boxes. In 17th Euromicro Conference on Digital System Design, DSD 2014, Verona, Italy, August 27-29, 2014, pages 512-519. IEEE Computer Society, 2014.

[WHS15] Markus Stefan Wamser, Lukas Holzbaur, and Georg Sigl. A petite and power saving design for the AES S-Box. In 2015 Euromicro Conference on Digital System Design, DSD 2015, Madeira, Portugal, August 26-28, 2015, pages 661-667. IEEE Computer Society, 2015.

[Wie99] Michael J. Wiener, editor. Advances in Cryptology - CRYPTO '99, 19th Annual International Cryptology Conference, Santa Barbara, California, USA, August 15-19, 1999, Proceedings, volume 1666 of Lecture Notes in Computer Science. Springer, 1999.

[WM12] Roy Ward and Timothy C.A. Molteno. Table of linear feedback shift registers. Technical Report 2012-1, University of Otago, 2012. http://www.physics . otago.ac.nz/reports/electronics/ETR2012-1.pdf.

[WM18] Felix Wegener and Amir Moradi. A first-order SCA resistant AES without fresh randomness. In Constructive Side-Channel Analysis and Secure Design - 9th International Workshop, COSADE 2018, Singapore, April 23-25, 2018, 2018.

[WS17] Markus Stefan Wamser and Georg Sigl. Pushing the limits further: Subatomic AES. In 2017 IFIP/IEEE International Conference on Very Large Scale Integration, VLSI-SoC 2017, Abu Dhabi, United Arab Emirates, October 23-25, 2017, pages 1-6. IEEE, 2017.

[Xil10] Xilinx. Spartan-6 FPGA configurable logic block user guide. https://www . xilinx.com/support/documentation/user_guides/ug384.pdf, 2010. 


\section{A ANFs for Byte-Serial Unprotected S-box}

The following results are valid in a normal basis with $\beta=145$. To allow replication of our results we share $S^{*}$ both as ANF and in a machine-readable notation (i.e. the 256-bit vector).

$$
\begin{aligned}
& \operatorname{rep}\left(S^{*}\right)=\text { 0x1c14813636f5767d6abc937b490334efd066cb1449f7ad147f30286c8bbef } 414 \\
& S^{*}(x)=x_{1} \oplus x_{2} \oplus x_{1} x_{3} \oplus x_{2} x_{3} \oplus x_{0} x_{2} x_{3} \oplus x_{1} x_{2} x_{3} \oplus x_{0} x_{1} x_{2} x_{3} \oplus x_{0} x_{4} \oplus x_{1} x_{4} \oplus x_{0} x_{1} x_{4} \\
& \oplus x_{2} x_{4} \oplus x_{0} x_{2} x_{4} \oplus x_{0} x_{1} x_{2} x_{4} \oplus x_{3} x_{4} \oplus x_{0} x_{3} x_{4} \oplus x_{0} x_{1} x_{3} x_{4} \oplus x_{0} x_{1} x_{2} x_{3} x_{4} \oplus x_{1} x_{5} \\
& \oplus x_{0} x_{1} x_{5} \oplus x_{0} x_{2} x_{5} \oplus x_{1} x_{2} x_{5} \oplus x_{0} x_{1} x_{3} x_{5} \oplus x_{0} x_{2} x_{3} x_{5} \oplus x_{2} x_{4} x_{5} \oplus x_{0} x_{2} x_{4} x_{5} \\
& \oplus x_{3} x_{4} x_{5} \oplus x_{0} x_{3} x_{4} x_{5} \oplus x_{1} x_{3} x_{4} x_{5} \oplus x_{0} x_{1} x_{3} x_{4} x_{5} \oplus x_{2} x_{3} x_{4} x_{5} \oplus x_{0} x_{2} x_{3} x_{4} x_{5} \\
& \oplus x_{1} x_{2} x_{3} x_{4} x_{5} \oplus x_{1} x_{6} \oplus x_{2} x_{6} \oplus x_{3} x_{6} \oplus x_{1} x_{3} x_{6} \oplus x_{0} x_{1} x_{3} x_{6} \oplus x_{0} x_{2} x_{3} x_{6} \\
& \oplus x_{0} x_{1} x_{2} x_{3} x_{6} \oplus x_{4} x_{6} \oplus x_{0} x_{4} x_{6} \oplus x_{1} x_{4} x_{6} \oplus x_{2} x_{4} x_{6} \oplus x_{0} x_{2} x_{4} x_{6} \oplus x_{1} x_{2} x_{4} x_{6} \\
& \oplus x_{0} x_{1} x_{2} x_{4} x_{6} \oplus x_{3} x_{4} x_{6} \oplus x_{0} x_{1} x_{3} x_{4} x_{6} \oplus x_{1} x_{2} x_{3} x_{4} x_{6} \oplus x_{1} x_{5} x_{6} \oplus x_{2} x_{5} x_{6} \oplus x_{3} x_{5} x_{6} \\
& \oplus x_{0} x_{3} x_{5} x_{6} \oplus x_{0} x_{1} x_{3} x_{5} x_{6} \oplus x_{1} x_{2} x_{3} x_{5} x_{6} \oplus x_{0} x_{1} x_{2} x_{3} x_{5} x_{6} \oplus x_{0} x_{4} x_{5} x_{6} \oplus x_{1} x_{4} x_{5} x_{6} \\
& \oplus x_{0} x_{2} x_{4} x_{5} x_{6} \oplus x_{1} x_{2} x_{4} x_{5} x_{6} \oplus x_{2} x_{3} x_{4} x_{5} x_{6} \oplus x_{1} x_{2} x_{3} x_{4} x_{5} x_{6} \oplus x_{0} x_{1} x_{2} x_{3} x_{4} x_{5} x_{6} \oplus x_{7} \\
& \oplus x_{0} x_{7} \oplus x_{1} x_{7} \oplus x_{0} x_{1} x_{7} \oplus x_{0} x_{2} x_{7} \oplus x_{1} x_{2} x_{7} \oplus x_{0} x_{1} x_{2} x_{7} \oplus x_{1} x_{3} x_{7} \oplus x_{2} x_{3} x_{7} \\
& \oplus x_{0} x_{2} x_{3} x_{7} \oplus x_{4} x_{7} \oplus x_{0} x_{4} x_{7} \oplus x_{3} x_{4} x_{7} \oplus x_{0} x_{1} x_{3} x_{4} x_{7} \oplus x_{1} x_{2} x_{3} x_{4} x_{7} \oplus x_{5} x_{7} \oplus x_{0} x_{5} x_{7} \\
& \oplus x_{0} x_{1} x_{5} x_{7} \oplus x_{2} x_{5} x_{7} \oplus x_{0} x_{2} x_{5} x_{7} \oplus x_{1} x_{2} x_{5} x_{7} \oplus x_{3} x_{5} x_{7} \oplus x_{0} x_{3} x_{5} x_{7} \oplus x_{2} x_{3} x_{5} x_{7} \\
& \oplus x_{0} x_{1} x_{2} x_{3} x_{5} x_{7} \oplus x_{1} x_{4} x_{5} x_{7} \oplus x_{0} x_{1} x_{4} x_{5} x_{7} \oplus x_{2} x_{4} x_{5} x_{7} \oplus x_{0} x_{2} x_{4} x_{5} x_{7} \oplus x_{0} x_{1} x_{2} x_{4} x_{5} x_{7} \\
& \oplus x_{0} x_{3} x_{4} x_{5} x_{7} \oplus x_{0} x_{1} x_{3} x_{4} x_{5} x_{7} \oplus x_{0} x_{2} x_{3} x_{4} x_{5} x_{7} \oplus x_{1} x_{2} x_{3} x_{4} x_{5} x_{7} \oplus x_{6} x_{7} \oplus x_{1} x_{6} x_{7} \\
& \oplus x_{0} x_{1} x_{6} x_{7} \oplus x_{2} x_{6} x_{7} \oplus x_{0} x_{2} x_{6} x_{7} \oplus x_{1} x_{2} x_{6} x_{7} \oplus x_{0} x_{3} x_{6} x_{7} \oplus x_{1} x_{3} x_{6} x_{7} \oplus x_{2} x_{3} x_{6} x_{7} \\
& \oplus x_{0} x_{2} x_{3} x_{6} x_{7} \oplus x_{1} x_{2} x_{3} x_{6} x_{7} \oplus x_{4} x_{6} x_{7} \oplus x_{1} x_{4} x_{6} x_{7} \oplus x_{2} x_{4} x_{6} x_{7} \oplus x_{0} x_{2} x_{4} x_{6} x_{7} \\
& \oplus x_{1} x_{2} x_{4} x_{6} x_{7} \oplus x_{0} x_{1} x_{2} x_{4} x_{6} x_{7} \oplus x_{0} x_{3} x_{4} x_{6} x_{7} \oplus x_{1} x_{3} x_{4} x_{6} x_{7} \oplus x_{2} x_{3} x_{4} x_{6} x_{7} \\
& \oplus x_{0} x_{2} x_{3} x_{4} x_{6} x_{7} \oplus x_{0} x_{5} x_{6} x_{7} \oplus x_{1} x_{5} x_{6} x_{7} \oplus x_{2} x_{5} x_{6} x_{7} \oplus x_{0} x_{2} x_{5} x_{6} x_{7} \oplus x_{3} x_{5} x_{6} x_{7} \\
& \oplus x_{0} x_{1} x_{2} x_{3} x_{5} x_{6} x_{7} \oplus x_{1} x_{4} x_{5} x_{6} x_{7} \oplus x_{2} x_{4} x_{5} x_{6} x_{7} \oplus x_{1} x_{3} x_{4} x_{5} x_{6} x_{7} \oplus x_{0} x_{1} x_{3} x_{4} x_{5} x_{6} x_{7} \\
& \oplus x_{2} x_{3} x_{4} x_{5} x_{6} x_{7}
\end{aligned}
$$

Furthermore, we provide the equations for the conversion from a polynomial base of $\operatorname{GF}\left(2^{8}\right)$ with $\alpha=2$ to a normal base with $\beta=145$ (p2n) and the conversion back concatenated with the affine function of the AES S-box (n2p).

$$
\begin{aligned}
& \mathrm{p} 2 \mathrm{n}_{0}(x)=x_{0} \oplus x_{1} \oplus x_{3} \oplus x_{6} \\
& \mathrm{p} 2 \mathrm{n}_{1}(x)=x_{0} \oplus x_{1} \oplus x_{2} \oplus x_{6} \oplus x_{7} \\
& \mathrm{p} 2 \mathrm{n}_{2}(x)=x_{0} \oplus x_{1} \oplus x_{2} \oplus x_{3} \oplus x_{4} \\
& \mathrm{p} 2 \mathrm{n}_{3}(x)=x_{0} \oplus x_{1} \oplus x_{2} \oplus x_{4} \oplus x_{6} \\
& \mathrm{p} 2 \mathrm{n}_{4}(x)=x_{0} \oplus x_{1} \oplus x_{2} \oplus x_{4} \oplus x_{5} \\
& \mathrm{p}^{2} \mathrm{n}_{5}(x)=x_{0} \oplus x_{1} \oplus x_{2} \oplus x_{4} \\
& \mathrm{p} 2 \mathrm{n}_{6}(x)=x_{0} \oplus x_{2} \oplus x_{3} \oplus x_{4} \\
& \mathrm{p} 2 \mathrm{n}_{7}(x)=x_{0} \oplus x_{3} \oplus x_{4} \oplus x_{6}
\end{aligned}
$$

$$
\begin{aligned}
& \mathrm{n} 2 \mathrm{p}_{0}(x)=x_{0} \oplus x_{1} \oplus x_{3} \oplus x_{4} \oplus x_{6} \oplus 1 \\
& \mathrm{n} 2 \mathrm{p}_{1}(x)=x_{1} \oplus x_{3} \oplus x_{4} \oplus x_{6} \oplus x_{7} \oplus 1 \\
& \mathrm{n} 2 \mathrm{p}_{2}(x)=x_{1}
\end{aligned}
$$




$$
\begin{aligned}
& \mathrm{n} 2 \mathrm{p}_{3}(x)=x_{1} \oplus x_{2} \oplus x_{3} \\
& \mathrm{n} 2 \mathrm{p}_{4}(x)=x_{2} \\
& \mathrm{n} 2 \mathrm{p}_{5}(x)=x_{0} \oplus x_{2} \oplus x_{3} \oplus x_{4} \oplus x_{5} \oplus x_{6} \oplus 1 \\
& \mathrm{n} 2 \mathrm{p}_{6}(x)=x_{0} \oplus x_{4} \oplus 1 \\
& \mathrm{n} 2 \mathrm{p}_{7}(x)=x_{1} \oplus x_{2} \oplus x_{4} \oplus x_{5}
\end{aligned}
$$

\section{B ANFs for Bit-Serial Unprotected S-box}

The following results are valid in a normal basis with $\beta=133$. To allow replication of our results we share $S^{*}$ both as $\mathrm{ANF}$ and in a machine-readable notation (i.e. the 256-bit vector).

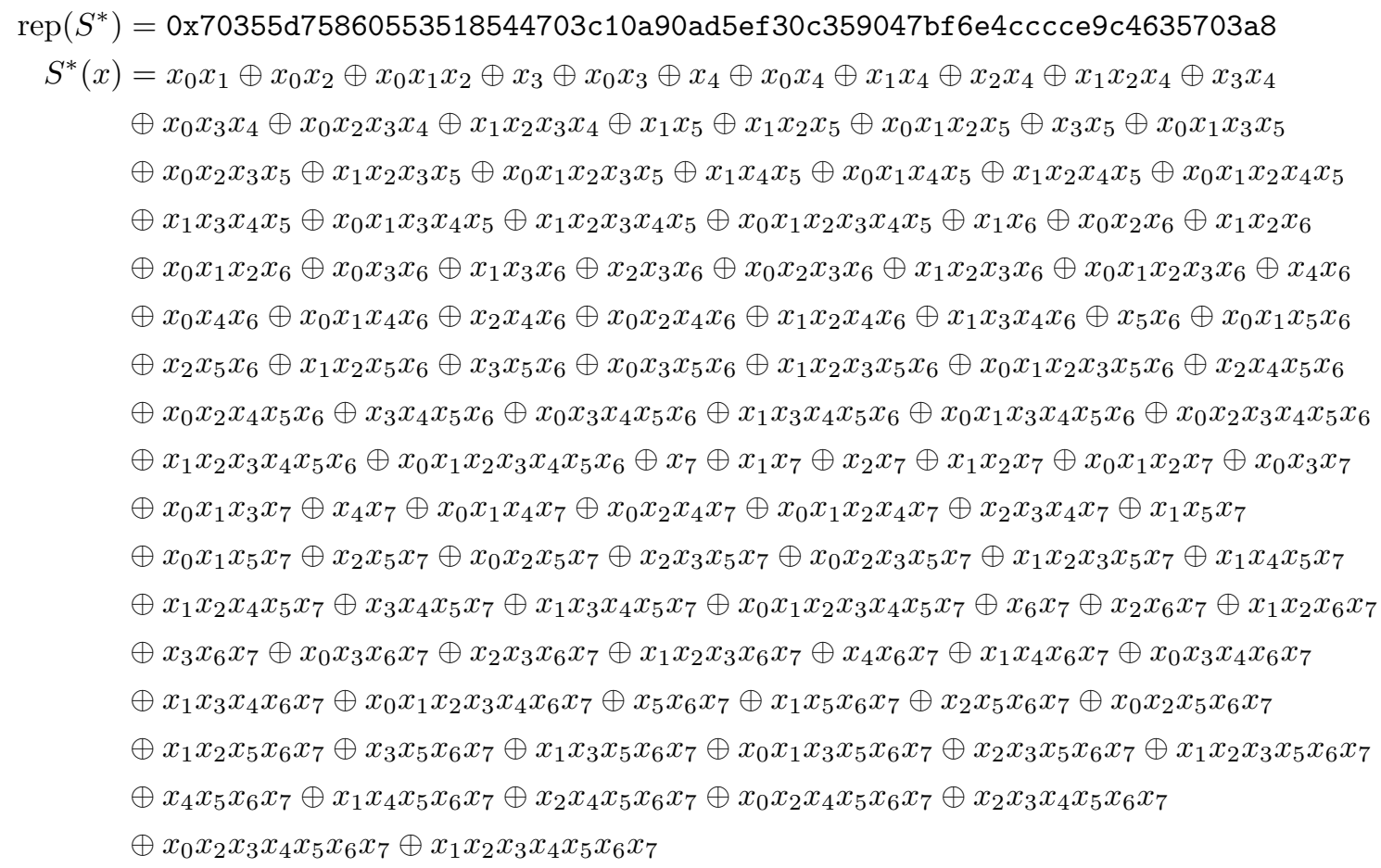

Furthermore, we provide the equations for the conversion from a polynomial base of $\mathrm{GF}\left(2^{8}\right)$ with $\alpha=2$ to a normal base with $\beta=133$ (p2n) and the conversion back concatenated with the affine function of the AES S-box (n2p).

$$
\begin{aligned}
& \mathrm{p} 2 \mathrm{n}_{0}(x)=x_{0} \oplus x_{3} \\
& \mathrm{p}^{2} \mathrm{n}_{1}(x)=x_{0} \oplus x_{1} \oplus x_{5} \oplus x_{6} \oplus x_{7} \\
& \mathrm{p} 2 \mathrm{n}_{2}(x)=x_{0} \oplus x_{2} \oplus x_{3} \oplus x_{5} \\
& \mathrm{p} 2 \mathrm{n}_{3}(x)=x_{0} \oplus x_{4} \oplus x_{6} \oplus x_{7} \\
& \mathrm{p} 2 \mathrm{n}_{4}(x)=x_{0} \oplus x_{1} \oplus x_{3} \oplus x_{5} \oplus x_{7} \\
& \mathrm{p}^{2} \mathrm{n}_{5}(x)=x_{0} \oplus x_{2} \oplus x_{3} \oplus x_{6} \\
& \mathrm{p}^{2} \mathrm{n}_{6}(x)=x_{0} \oplus x_{4} \oplus x_{5} \oplus x_{6} \oplus x_{7} \\
& \mathrm{p} 2 \mathrm{n}_{7}(x)=x_{0} \oplus x_{5} \oplus x_{7}
\end{aligned}
$$




$$
\begin{aligned}
& \mathrm{n} 2 \mathrm{p}_{0}(x)=x_{6} \oplus 1 \\
& \mathrm{n} 2 \mathrm{p}_{1}(x)=x_{1} \oplus 1 \\
& \mathrm{n} 2 \mathrm{p}_{2}(x)=x_{0} \oplus x_{1} \oplus x_{2} \\
& \mathrm{n} 2 \mathrm{p}_{3}(x)=x_{0} \oplus x_{2} \oplus x_{4} \\
& \mathrm{n} 2 \mathrm{p}_{4}(x)=x_{1} \oplus x_{2} \oplus x_{3}
\end{aligned}
$$

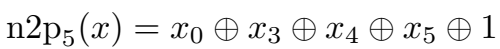

$$
\begin{aligned}
& \mathrm{n} 2 \mathrm{p}_{6}(x)=x_{0} \oplus x_{1} \oplus x_{3} \oplus x_{4} \oplus x_{5} \oplus x_{7} \oplus 1 \\
& \mathrm{n} 2 \mathrm{p}_{7}(x)=x_{0} \oplus x_{6}
\end{aligned}
$$

\section{ANFs for Masked S-box}

The following 3 -splits are valid in a normal basis with $\beta=205$. We designate by $x_{\bar{i}}$ all bits of $x$ except the $i^{\text {th }}$,i.e. $\left(x_{0}, \ldots, x_{i-1}, x_{i+1}, \ldots, x_{7}\right)$

$$
\begin{aligned}
G^{A}\left(x_{\overline{5}}\right) & =x_{0} x_{3} x_{7} \oplus x_{0} x_{4} x_{6} \oplus x_{0} x_{4} x_{2} \oplus x_{0} x_{3} x_{2} \oplus x_{1} x_{4} x_{7} \oplus x_{1} x_{3} x_{7} \oplus x_{1} x_{4} x_{2} \oplus x_{1} x_{3} x_{2} \\
& \oplus x_{0} x_{6} \oplus x_{0} x_{4} \oplus x_{0} x_{2} \oplus x_{3} x_{7} \oplus x_{1} x_{7} \oplus x_{4} x_{2} \oplus x_{1} x_{3} \oplus x_{1} x_{2} \oplus x_{4} \oplus x_{6} \\
G^{B}\left(x_{\overline{0}}\right) & =x_{1} x_{2} x_{6} \oplus x_{1} x_{4} x_{3} \oplus x_{1} x_{4} x_{5} \oplus x_{7} x_{2} x_{3} \oplus x_{7} x_{2} x_{5} \oplus x_{7} x_{4} x_{5} \oplus x_{7} x_{2} x_{6} \oplus x_{7} x_{4} x_{6} \\
& \oplus x_{7} x_{5} \oplus x_{4} x_{5} \oplus x_{2} x_{5} \\
G^{C}\left(x_{\overline{3}}\right) & =x_{0} x_{1} x_{5} \oplus x_{0} x_{1} x_{2} \oplus x_{7} x_{4} x_{2} \oplus x_{6} x_{4} x_{5} \oplus x_{6} x_{1} x_{5} \oplus x_{0} x_{5}
\end{aligned}
$$

$(F \oplus G)^{A}\left(x_{\overline{6}}\right)=x_{0} x_{1} x_{3} \oplus x_{0} x_{1} x_{4} \oplus x_{0} x_{5} x_{4} \oplus x_{2} x_{5} x_{4} \oplus x_{7} x_{1} x_{3} \oplus x_{7} x_{5} x_{3} \oplus x_{7} x_{5} x_{4} \oplus x_{2} x_{5}$ $\oplus x_{5} x_{3} \oplus x_{5} x_{4} \oplus x_{7} x_{3} \oplus x_{7} x_{4} \oplus x_{7} x_{5} \oplus x_{0} x_{1} \oplus x_{0} x_{5} \oplus x_{0} \oplus x_{4}$

$(F \oplus G)^{B}\left(x_{\overline{6}}\right)=x_{0} x_{2} x_{3} \oplus x_{7} x_{2} x_{3} \oplus x_{7} x_{2} x_{5} \oplus x_{1} x_{2} x_{5} \oplus x_{1} x_{4} x_{5} \oplus x_{1} x_{4} x_{3} \oplus x_{4} x_{3}$

$(F \oplus G)^{C}\left(x_{\overline{1}}\right)=x_{0} x_{4} x_{7} \oplus x_{0} x_{2} x_{7} \oplus x_{0} x_{2} x_{6} \oplus x_{3} x_{4} x_{6} \oplus x_{3} x_{5} x_{6} \oplus x_{3} x_{2} x_{6} \oplus x_{0} x_{6} \oplus x_{4} x_{6}$

To allow a convenient replication of our results we additionally provide the functions in a machine-readable notation (i.e. the 256 -bit vector).

$$
\begin{aligned}
\operatorname{rep}\left(G^{A}\right) & =0 \mathrm{x} 000000000000000000000000000040704000000000002000300000000000736460 \\
\operatorname{rep}\left(G^{B}\right) & =0 \mathrm{x} 00000000000100100001001100001000000000000000004000050010040000000 \\
\operatorname{rep}\left(G^{C}\right) & =0 \mathrm{x} 0000000000000000000000000010000000010004000000000000000 \mathrm{a} 00000080 \\
\operatorname{rep}\left((F \oplus G)^{A}\right) & =0 \mathrm{x} 000000000000000000010101000105000000000000000000001301120009080 \mathrm{a} \\
\operatorname{rep}\left((F \oplus G)^{B}\right) & =0 \mathrm{x} 0000000000000000000000100000100000000000000000000004004005002000 \\
\operatorname{rep}\left((F \oplus G)^{C}\right) & =0 \mathrm{x} 00000000000000000000000000020020000001000101102200000000000000000
\end{aligned}
$$

Furthermore, we provide the equations for the conversion from a polynomial base of $\operatorname{GF}\left(2^{8}\right)$ with $\alpha=2$ to a normal base with $\beta=205(\mathrm{p} 2 \mathrm{n})$ and the conversion back concatenated with the affine function of the AES S-box (n2p).

$$
\begin{aligned}
& \mathrm{p} 2 \mathrm{n}_{0}(x)=x_{0} \oplus x_{2} \oplus x_{7} \\
& \mathrm{p} 2 \mathrm{n}_{1}(x)=x_{2} \oplus x_{6} \\
& \mathrm{p} 2 \mathrm{n}_{2}(x)=x_{0} \oplus x_{2} \oplus x_{3} \oplus x_{4} \oplus x_{5} \oplus x_{7}
\end{aligned}
$$




$$
\begin{aligned}
& \mathrm{p} 2 \mathrm{n}_{3}(x)=x_{0} \oplus x_{4} \oplus x_{5} \oplus x_{6} \\
& \mathrm{p} 2 \mathrm{n}_{4}(x)=x_{4} \oplus x_{7} \\
& \mathrm{p} 2 \mathrm{n}_{5}(x)=x_{1} \oplus x_{3} \oplus x_{4} \oplus x_{7} \\
& \mathrm{p} 2 \mathrm{n}_{6}(x)=x_{0} \oplus x_{1} \oplus x_{2} \oplus x_{4} \oplus x_{6} \oplus x_{7} \\
& \mathrm{p} 2 \mathrm{n}_{7}(x)=x_{0} \oplus x_{2} \oplus x_{5} \oplus x_{6} \\
& \mathrm{n} 2 \mathrm{p}_{0}(x)=x_{0} \oplus x_{2} \oplus x_{3} \oplus x_{4} \oplus x_{5} \oplus 1 \\
& \mathrm{n} 2 \mathrm{p}_{1}(x)=x_{0} \oplus x_{3} \oplus x_{5} \oplus x_{6} \oplus x_{7} \oplus 1 \\
& \mathrm{n} 2 \mathrm{p}_{2}(x)=x_{1} \oplus x_{2} \oplus x_{3} \oplus x_{6} \oplus x_{7} \\
& \mathrm{n} 2 \mathrm{p}_{3}(x)=x_{3} \oplus x_{5} \oplus x_{6} \oplus \\
& \mathrm{n} 2 \mathrm{p}_{4}(x)=x_{0} \oplus x_{2} \oplus x_{3} \oplus x_{4} \oplus x_{7} \\
& \mathrm{n} 2 \mathrm{p}_{5}(x)=x_{1} \oplus x_{7} \oplus 1 \\
& \mathrm{n} 2 \mathrm{p}_{6}(x)=x_{0} \oplus x_{4} \oplus 1 \\
& \mathrm{n} 2 \mathrm{p}_{7}(x)=x_{0} \oplus x_{3} \oplus x_{6} \oplus x_{7}
\end{aligned}
$$

